\title{
Synthesis, characterization and in vitro biological evaluation of some new diarylsulfonylurea-chalcone hybrids as potential 5-lipoxygenase inhibitors
}

\author{
Bharat Kumar Bugata a,*, Satya Venkata Gopala Krishna Kaladhar Dowluru a, Vasudeva Rao Avupati b, \\ Venkateswara Rao Gavalapu b, Divakara Laxman Somayajulu Nori c and Sreenu Barla a \\ a Department of Biochemistry and Bioinformatics, Gitam University, Rushikonda, Visakhapatnam-530045, Andhra Pradesh, India \\ b Pharmaceutical Chemistry Division, Andhra University College of Pharmaceutical Sciences, Andhra University, Visakhapatnam-530003, Andhra Pradesh, India \\ c Department of Chemistry, Gitam University, Rushikonda, Visakhapatnam-530045, Andhra Pradesh, India \\ ${ }^{*}$ Corresponding author at: Department of Biochemistry and Bioinformatics, Gitam University, Rushikonda, Visakhapatnam-530045, Andhra Pradesh, India. \\ Tel.: +91.0891.2792928; fax: +91.0891.2790399. E-mail address: bharat8891@gmail.com (B.K. Bugata).
}

\section{ARTICLE INFORMATION}

Received: 15 July 2013

Received in revised form: 04 August 2013

Accepted: 06 August 2013

Online: 31 December 2013

\section{KEYWORDS}

\section{Chalcone}

5-Lipoxygenase

Diarylsulfonylurea

Abietic acid (LI01020)

Claisen-Schmidt condensation

Diarylsulfonylurea-chalcone hybrid

\section{Introduction}

Lipoxygenases are a class of non-heme, iron-containing enzymes that catalyze the incorporation of molecular oxygen into 1,4,-cis,cis-pentadiene-containing fatty acids (e.g. linoleic and arachidonic acids) to form hydroperoxide products [1]. The human isozymes, 5-, 12- and 15-Lipoxygenases are associated with different disease states, which suggests that selective inhibition may be important in targeting them for therapeutic purposes. 5-Lipoxygenase (5-LO), which was first discovered in 1976, plays an essential role in the biosynthesis of leukotrienes (LTs) that exert a large number of different biological activities mediated by specific G-protein coupled receptors. $\mathrm{LTB}_{4}$ is a typical proinflammatory mediator that recruits and activates leukocytes, whereas cysteinyl-leukotrienes $\mathrm{C}_{4}, \mathrm{D}_{4}$ and $\mathrm{E}_{4}$ cause vascular permeability and smooth muscle contraction. In view of these properties, development of drugs with 5-LO inhibitory activity has been hypothesized to possess therapeutic potential for treatment of asthma, allergic disorders and other inflammatory diseases [2]. Based on the mechanism of action, the lipoxygenase inhibitors have been classified into four distinct classes:

(i) Iron chelating inhibitors,

(ii) Competitive reversible inhibitors,

(iii) Inhibitors of the 5-LO activating protein (FLAP) and

(iv) Anti-oxidative [3]. Intensive discovery efforts in the development of clinically useful drugs from the inhibitors of $5 \mathrm{LO}$ enzyme have led to one marketed drug; Zileuton (A-64066) and others, namely MK-3000, MK-886, MK0591, ZM 211965, AKBA, BW A4C, LDP-977, Bay-X-1005, and Abt-761, which are evaluated at different stages of drug development $[2,3]$.

European Journal of Chemistry

ISSN 2153-2249 (Print) / ISSN 2153-2257 (Online) @ 2013 EURJCHEM

DOI:10.5155/eurjchem.4.4.396-401.878
Diarylsulfonylureas are the structural analogs of urea $\left(\mathrm{NH}_{2} \mathrm{CONH}_{2}\right)$ with aromatic sulfonyl group in the position 3 and an aromatic or heteroaromatic ring at the position 1 . Diarylsulfonylureas became widely available since 1955 as popular antidiabetic drugs in clinical practice for the treatment of type 2 diabetes, by virtue of their insulin secretagogue properties. The synthesis of compounds containing diarylsulfonylurea moiety has been a subject of extensive research in the recent past because of their enormous biological activities such as hypoglycemics [4], Vibrio fischeri quorum sensing regulators [5], CXCR2 receptor antagonists [6], antimalarials [7], antibacterials [8], human thromboxane $A_{2}$ receptor isoforms $\mathrm{TP}_{\alpha}$ and $\mathrm{TP}_{\beta}$ antagonists [9], reversible inhibitors of human steroid sulfatase [10], Katp-channel openers [11], ANG II ( $\left.\mathrm{AT}_{1}\right)$ receptor antagonists [12], oncolytics [13], acyl-CoA inhibitors [14], vasodilators [15], aldehyde dehydrogenase inhibitors [16], cancer chemotherapeutics [17], diuretic [18], $\beta_{3}$ adrenergic receptor agonists [19], non competitive inhibitors of acetohydroxyacid synthase from Mycobacterium tuberculosis [20], and as peroxisome proliferator activated receptor gamma (PPAR $\gamma$ ) agonists [21].

Similarly, chalcones ( $\alpha, \beta$-unsatured ketones) have also been gained huge significance as these compounds exhibit several biological activities, such as antimicrobial [22], antiviral [23], antioxidant [24], radical inhibitor [25], antitumor [26], carbonic anhydrase inhibitor [27], xanthine oxidase inhibitor [28], antibacterial [29], plant growth regulator [30], free radical scavenger [31], anti-inflammatory [32] and analgesic [33]. These activities are largely attributed due to the $\alpha, \beta$-unsatured ketone moiety [34]. Consequently a number of strategies have been originated to synthesize them [35-37]. 


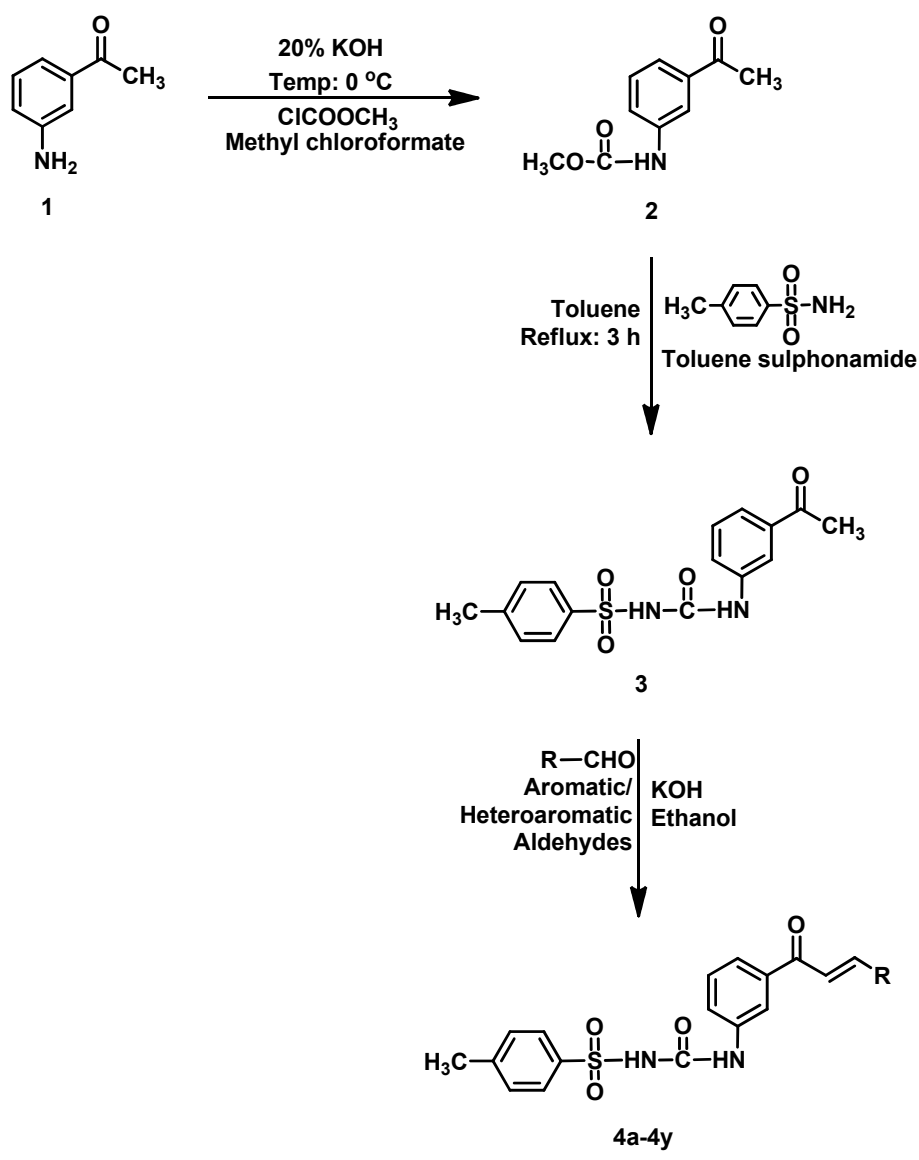

Scheme 1

Based on the above observations, an attempt has been made in the present study to combine these two bioactive pharmacophores in a single molecular platform through molecular hybridization strategies. Hence, it was considered worthwhile to synthesize and characterize some novel diarylsulfonylurea-chalcone hybrids $(\mathbf{4 a - 4 y )}$ in the present study [38]. To the best of our knowledge there is, to date, no report that diarylsulfonylurea-chalcone hybrid derivatives have any inhibitory activity against 5-LO.

\section{Experimental}

\subsection{Instrumentation}

Melting points were taken in open capillary tubes and are therefore uncorrected. Purity of the compounds was checked on silica gel G TLC plates of $2 \mathrm{~mm}$ thickness using $n$-hexane and ethyl acetate as solvent system. The visualization of spot was carried out in an iodine chamber. The FT-IR spectra were recorded on Perkin-Elmer spectrometer. The ${ }^{1} \mathrm{H}$ NMR spectra were scanned on a Bruker $400 \mathrm{MHz}$. spectrometer in DMSO- $d_{6}$ using TMS as internal standard and chemical shifts are expressed in $\delta \mathrm{ppm}$. The ESI mass spectra were recorded on an Agilent 6100 QQQ spectrometer (positive ion mode).

\subsection{General procedure for the synthesis of diarylsulfonylurea-chalcone hybrids $(4 a-4 y)$}

The reaction sequence employed in the synthesis of diarylsulfonylurea-chalcone hybrids (4a-4y) is shown in the Scheme 1. The key intermediates in the present study (2) and
(3) were synthesized from (1) as reported earlier [33,34]. Subsequent Claisen-Schmidt condensation [39] of the intermediate 1-(3-acetylphenyl)-3-tosylurea (3) with appropriate aromatic/heteroaromatic aldehydes in ethanolic $\mathrm{KOH}$ solution $(100 \%)$ to give the corresponding diarylsulfonylurea-chalcone hybrids (4a-4y) in good yield. All the structures of the compounds were appropriately established by spectroscopic data and analytical methods.

(E)-1-[3-(3-(phenyl) acryloyl) phenyl]-3-tosylurea (4a): Colour: Yellow. Yield: $97 \%$. M.p.: $151.3{ }^{\circ}$ C. FT-IR $\left(\mathrm{KBr}, v_{\max }, \mathrm{cm}\right.$ 1): 3317, $3314(\mathrm{~N}-\mathrm{H}), 3011(\mathrm{C}-\mathrm{H}), 1656(\mathrm{C}=0), 1621(\mathrm{C}=\mathrm{C})$, 1555 (CONH), 1549 (N-H bend), 1300 ( $\mathrm{SO}_{2}$, asym.), 1329 (C-N), $1120\left(\mathrm{SO}_{2}\right.$, sym.). ${ }^{1} \mathrm{H}$ NMR $\left(400 \mathrm{MHz}\right.$, DMSO- $\left.d_{6}, \delta, \mathrm{ppm}\right): 2.30(\mathrm{~s}$, $\left.3 \mathrm{H}, \mathrm{CH}_{3}\right), 7.41-7.59(\mathrm{~m}, 9 \mathrm{H}, \mathrm{Ar}-\mathrm{H}), 7.42(\mathrm{~d}, J=8.1 \mathrm{~Hz}, 2 \mathrm{H}, \mathrm{Ar}-\mathrm{H})$, $7.65(\mathrm{~d}, J=15.2 \mathrm{~Hz}, 1 \mathrm{H}, \mathrm{HC}=\mathrm{CH}(\mathrm{H}-\alpha)), 7.96(\mathrm{~d}, J=15.2 \mathrm{~Hz}, 1 \mathrm{H}$, $\mathrm{HC}=\mathrm{CH}(\mathrm{H}-\beta)), 8.07(\mathrm{~d}, J=8.1 \mathrm{~Hz}, 2 \mathrm{H}, \mathrm{Ar}-\mathrm{H}), 10.33(\mathrm{~s}, 1 \mathrm{H}, \mathrm{NH})$, $11.73(\mathrm{~s}, 1 \mathrm{H}, \mathrm{NH})$. ESI-MS $(\mathrm{m} / \mathrm{z}): 421[\mathrm{M}+\mathrm{H}]^{+}$. Anal. calcd. for $\mathrm{C}_{23} \mathrm{H}_{20} \mathrm{~N}_{2} \mathrm{O}_{4} \mathrm{~S}: \mathrm{C}, 65.42 ; \mathrm{H}, 4.33 ; \mathrm{N}, 6.35$. Found: $\mathrm{C}, 65.70 ; \mathrm{H}, 4.79$; $\mathrm{N}, 6.66 \%$.

(E)-1-[3-(3-(tolyl) acryloyl) phenyl]-3-tosylurea (4b): Colour: Yellow. Yield: $89 \%$. M.p.: $233.8^{\circ} \mathrm{C}$. FT-IR $\left(\mathrm{KBr}, v_{\max }, \mathrm{cm}^{-}\right.$ 1): 3428, $3317(\mathrm{~N}-\mathrm{H}), 3073(\mathrm{C}-\mathrm{H}), 1645(\mathrm{C}=\mathrm{O}), 1616(\mathrm{C}=\mathrm{C})$, 3073 (C-H), 1575 (CONH), 1337 (C-N), 1542 (N-H bend), 1337 (C-N) 1298 ( $\mathrm{SO}_{2}$, asym.), 1182 ( $\mathrm{SO}_{2}$, sym.). ${ }^{1} \mathrm{H}$ NMR $(400 \mathrm{MHz}$, DMSO- $\left.d_{6}, \delta, \mathrm{ppm}\right): 2.30\left(\mathrm{~s}, 3 \mathrm{H}, \mathrm{CH}_{3}\right), 2.34\left(\mathrm{~s}, 3 \mathrm{H}, \mathrm{CH}_{3}\right), 7.17-7.38$ $(\mathrm{m}, 8 \mathrm{H}, \mathrm{Ar}-\mathrm{H}), 7.42(\mathrm{~d}, J=8.1 \mathrm{~Hz}, 2 \mathrm{H}, \mathrm{Ar}-\mathrm{H}), 7.62(\mathrm{~d}, J=15.2 \mathrm{~Hz}$, $1 \mathrm{H}, \mathrm{HC}=\mathrm{CH}(\mathrm{H}-\alpha)), 7.91(\mathrm{~d}, J=15.2 \mathrm{~Hz}, 1 \mathrm{H}, \mathrm{HC}=\mathrm{CH}(\mathrm{H}-\beta)), 8.07$ (d, $J=8.1 \mathrm{~Hz}, 2 \mathrm{H}, \mathrm{Ar}-\mathrm{H}), 10.33(\mathrm{~s}, 1 \mathrm{H}, \mathrm{NH}), 11.73(\mathrm{~s}, 1 \mathrm{H}, \mathrm{NH})$. ESI-MS $(m / z): 435[\mathrm{M}+\mathrm{H}]^{+}$. Anal. calcd. for $\mathrm{C}_{24} \mathrm{H}_{22} \mathrm{~N}_{2} \mathrm{O}_{4} \mathrm{~S}$ : C, $66.34 ; \mathrm{H}, 5.10 ; \mathrm{N}, 6.45$. Found: C, 66.32; H, 5.13; N, 6.55\%. 
(E)-1-[3-(3-(4-N,N-dimethylaminophenyl) acryloyl) phenyl]3-tosylurea (4c): Colour: Yellow. Yield: 88\%. M.p.: $150.5{ }^{\circ} \mathrm{C}$. FTIR (KBr, $\left.v_{\max }, \mathrm{cm}^{-1}\right): 3460(\mathrm{~N}-\mathrm{H}), 3341(\mathrm{C}=0), 3038(\mathrm{C}-\mathrm{H}), 1651$ $(\mathrm{C}=\mathrm{C}), 1591(\mathrm{CONH}), 1531$ (N-H bend), $1353(\mathrm{C}-\mathrm{N}), 1310\left(\mathrm{SO}_{2}\right.$, asym.), $1156\left(\mathrm{SO}_{2}\right.$, sym.). ${ }^{1} \mathrm{H}$ NMR $\left(400 \mathrm{MHz}\right.$, DMSO- $\left.d_{6}, \delta, \mathrm{ppm}\right)$ : $2.30\left(\mathrm{~s}, 3 \mathrm{H}, \mathrm{CH}_{3}\right), 2.38$ (s, 6H, $\left.\mathrm{CH}_{3}\right), 7.36-7.77(\mathrm{~m}, 8 \mathrm{H}, \mathrm{Ar}-\mathrm{H}), 7.43$ $(\mathrm{d}, J=8.1 \mathrm{~Hz}, 2 \mathrm{H}, \mathrm{Ar}-\mathrm{H}), 7.69(\mathrm{~d}, J=15.2 \mathrm{~Hz}, 1 \mathrm{H}, \mathrm{HC}=\mathrm{CH}(\mathrm{H}-\alpha))$, $7.84(\mathrm{~d}, J=15.2 \mathrm{~Hz}, 1 \mathrm{H}, \mathrm{HC}=\mathrm{CH}(\mathrm{H}-\beta)), 8.01(\mathrm{~d}, J=8.1 \mathrm{~Hz}, 2 \mathrm{H}$, Ar-H), 10.89 (s, 1H, NH), 12.02 (s, 1H, NH). ESI-MS $(\mathrm{m} / \mathrm{z}): 464$ $[\mathrm{M}+\mathrm{H}]^{+}$. Anal. calcd. for $\mathrm{C}_{25} \mathrm{H}_{25} \mathrm{~N}_{3} \mathrm{O}_{4} \mathrm{~S}: \mathrm{C}, 64.78 ; \mathrm{H}, 5.44 ; \mathrm{N}, 9.06$. Found: C, 64.71; H, 5.25; N, 9.11\%.

(E)-1-[3-(3-(2,4-dimethoxyphenyl) acryloyl) phenyl]-3tosylurea (4d): Colour: Yellow. Yield: 84\%. M.p.: $163.3^{\circ} \mathrm{C}$. FT-IR $\left(\mathrm{KBr}, v_{\max }, \mathrm{cm}^{-1}\right): 3225(\mathrm{~N}-\mathrm{H}), 2888(\mathrm{C}-\mathrm{H}), 1716(\mathrm{C}=0), 1655$ $(\mathrm{C}=\mathrm{C}), 1593(\mathrm{CONH}), 1528\left(\mathrm{~N}-\mathrm{H}\right.$ bend), $1356(\mathrm{C}-\mathrm{N}), 1312\left(\mathrm{SO}_{2}\right.$, asym.), 1153 ( $\mathrm{SO}_{2}$, sym.). ${ }^{1} \mathrm{H}$ NMR ( $400 \mathrm{MHz}$, DMSO- $\left.d_{6}, \delta, \mathrm{ppm}\right)$ : $2.30\left(\mathrm{~s}, 3 \mathrm{H}, \mathrm{CH}_{3}\right), 3.85\left(\mathrm{~s}, 6 \mathrm{H}, \mathrm{OCH}_{3}\right), 7.41(\mathrm{~d}, J=8.1 \mathrm{~Hz}, 2 \mathrm{H}, \mathrm{Ar}-$ H), 7.66-7.98 (m, 7H, Ar-H), 7.69 (d, $J=15.2 \mathrm{~Hz}, 1 \mathrm{H}, \mathrm{HC}=\mathrm{CH}$ $(\mathrm{H}-\alpha)), 7.85(\mathrm{~d}, J=8.1 \mathrm{~Hz}, 2 \mathrm{H}, \mathrm{Ar}-\mathrm{H}), 7.98(\mathrm{~d}, J=15.2 \mathrm{~Hz}, 1 \mathrm{H}$, $\mathrm{HC}=\mathrm{CH}(\mathrm{H}-\beta)), 9.01(\mathrm{~s}, 1 \mathrm{H}, \mathrm{NH}), 10.98(\mathrm{~s}, 1 \mathrm{H}, \mathrm{NH})$. ESI-MS $(\mathrm{m} / \mathrm{z}): 481[\mathrm{M}+\mathrm{H}]^{+}$. Anal. calcd. for $\mathrm{C}_{25} \mathrm{H}_{24} \mathrm{~N}_{2} \mathrm{O}_{6} \mathrm{~S}$ : C, 62.49; $\mathrm{H}$, 5.03; N, 5.83. Found: C, 62.23; H, 5.16; N, 5.78\%.

(E)-1-[3-(3-(3,4,5-trimethoxyphenyl) acryloyl) phenyl]-3tosylurea (4e): Colour: Yellow. Yield: 88\%. M.p.: $198.6^{\circ} \mathrm{C}$. FT-IR (KBr, $\left.v_{\max }, \mathrm{cm}^{-1}\right): 3355,3305(\mathrm{~N}-\mathrm{H}), 2976(\mathrm{C}-\mathrm{H}), 1595(\mathrm{C}=0)$, $1516(\mathrm{C}=\mathrm{C}), 1471(\mathrm{CONH}), 1441$ (N-H bend), 1307 (C-N), 1280 ( $\mathrm{SO}_{2}$, asym.), 1135 ( $\mathrm{SO}_{2}$, sym.). ${ }^{1} \mathrm{H}$ NMR $\left(400 \mathrm{MHz}, \mathrm{DMSO}-d_{6}, \delta\right.$, ppm): 2.30 (s, $\left.3 \mathrm{H}, \mathrm{CH}_{3}\right), 3.71\left(\mathrm{~s}, 3 \mathrm{H}, \mathrm{OCH}_{3}\right), 3.85\left(\mathrm{~s}, 6 \mathrm{H}, \mathrm{OCH}_{3}\right)$, $7.25(\mathrm{~d}, J=8.1 \mathrm{~Hz}, 2 \mathrm{H}, \mathrm{Ar}-\mathrm{H}), 7.43-7.77(\mathrm{~m}, 6 \mathrm{H}, \mathrm{Ar}-\mathrm{H}), 7.69(\mathrm{~d}, J$ $=15.2 \mathrm{~Hz}, 1 \mathrm{H}, \mathrm{HC}=\mathrm{CH}(\mathrm{H}-\alpha)), 7.85(\mathrm{~d}, J=8.1 \mathrm{~Hz}, 2 \mathrm{H}, \mathrm{Ar}-\mathrm{H}), 7.98$ (d, $J=15.2 \mathrm{~Hz}, 1 \mathrm{H}, \mathrm{HC}=\mathrm{CH}(\mathrm{H}-\beta)), 8.92(\mathrm{~s}, 1 \mathrm{H}, \mathrm{NH}), 10.41(\mathrm{~s}, 1 \mathrm{H}$, NH). ESI-MS $(m / z): 511[\mathrm{M}+\mathrm{H}]^{+}$. Anal. calcd. for $\mathrm{C}_{26} \mathrm{H}_{26} \mathrm{~N}_{2} \mathrm{O}_{7} \mathrm{~S}: \mathrm{C}$, 61.16; H, 5.13; N, 5.49. Found: C, 61.22; H, 5.21; N, 5.51\%.

(E)-1-[3-(3-(2-hydroxyphenyl) acryloyl) phenyl]-3-tosylurea (4f): Colour: Yellow. Yield: 86\%. M.p.: 258.9 oC. FT-IR (KBr, $v_{\max }$, $\left.\mathrm{cm}^{-1}\right)$ : $3225(\mathrm{~N}-\mathrm{H}), 1716(\mathrm{C}=0), 1655(\mathrm{C}=\mathrm{C}), 1592(\mathrm{C}-\mathrm{H}), 1528$ (N-H bend), 1449 (CONH), 1341 (C-N), 1300 ( $\mathrm{SO}_{2}$, asym.), 1153 ( $\mathrm{SO}_{2}$, sym.). ${ }^{1} \mathrm{H}$ NMR $\left(400 \mathrm{MHz}, \mathrm{DMSO}-d_{6}, \delta, \mathrm{ppm}\right): 2.30$ (s, 3H, $\left.\mathrm{CH}_{3}\right), 7.37-7.93(\mathrm{~m}, 8 \mathrm{H}, \mathrm{Ar}-\mathrm{H}), 7.47(\mathrm{~d}, J=8.1 \mathrm{~Hz}, 2 \mathrm{H}, \mathrm{Ar}-\mathrm{H}), 7.70$ $(\mathrm{d}, J=15.4 \mathrm{~Hz}, 1 \mathrm{H}, \mathrm{HC}=\mathrm{CH}(\mathrm{H}-\alpha)), 7.81(\mathrm{~d}, J=8.1 \mathrm{~Hz}, 2 \mathrm{H}, \mathrm{Ar}-\mathrm{H})$, $7.88(\mathrm{~d}, J=15.4 \mathrm{~Hz}, 1 \mathrm{H}, \mathrm{HC}=\mathrm{CH}(\mathrm{H}-\beta)), 10.12(\mathrm{~s}, 1 \mathrm{H}, \mathrm{OH}), 10.64$ (s, 1H, NH); $11.98(\mathrm{~s}, 1 \mathrm{H}, \mathrm{NH})$. ESI-MS $(\mathrm{m} / \mathrm{z}): 437[\mathrm{M}+\mathrm{H}]^{+}$. Anal. calcd. for $\mathrm{C}_{23} \mathrm{H}_{20} \mathrm{~N}_{2} \mathrm{O}_{5} \mathrm{~S}$ : C, 63.29; $\mathrm{H}, 4.62 ; \mathrm{N}, 6.42$. Found: $\mathrm{C}$, 63.33; $\mathrm{H}, 4.71 ; \mathrm{N}, 6.55 \%$.

(E)-1-[3-(3-(3-hydroxyphenyl) acryloyl) phenyl]-3-tosylurea (4g): Colour: Yellow. Yield: 82\%. M.p.: $185.2{ }^{\circ} \mathrm{C}$. FT-IR (KBr, $\left.V_{\max } \mathrm{cm}^{-1}\right): 3226(\mathrm{~N}-\mathrm{H}), 1716(\mathrm{C}=0), 1655(\mathrm{C}=\mathrm{C}), 1592(\mathrm{C}-\mathrm{H})$, 1528 ( N-H bend), 1449 (CONH), 1341 (C-N), 1300 ( $\mathrm{SO}_{2}$, asym.), $1153\left(\mathrm{SO}_{2}\right.$, sym.). ${ }^{1} \mathrm{H}$ NMR $\left(400 \mathrm{MHz}\right.$, DMSO- $\left.d_{6}, \delta, \mathrm{ppm}\right): 2.30$ (s, $\left.3 \mathrm{H}, \mathrm{CH}_{3}\right), 7.22-7.63(\mathrm{~m}, 8 \mathrm{H}, \mathrm{Ar}-\mathrm{H}), 7.42(\mathrm{~d}, J=8.1 \mathrm{~Hz}, 2 \mathrm{H}, \mathrm{Ar}-\mathrm{H})$, $7.45(\mathrm{~s}, 1 \mathrm{H}, \mathrm{OH}), 7.69(\mathrm{~d}, J=15.4 \mathrm{~Hz}, 1 \mathrm{H}, \mathrm{HC}=\mathrm{CH}(\mathrm{H}-\alpha)), 7.81(\mathrm{~d}$, $J=15.4 \mathrm{~Hz}, 1 \mathrm{H}, \mathrm{HC}=\mathrm{CH}(\mathrm{H}-\beta)), 7.83(\mathrm{~d}, J=8.1 \mathrm{~Hz}, 2 \mathrm{H}, \mathrm{Ar}-\mathrm{H})$, $10.87(\mathrm{~s}, 1 \mathrm{H}, \mathrm{NH}), 12.12(\mathrm{~s}, 1 \mathrm{H}, \mathrm{NH})$. ESI-MS $(\mathrm{m} / \mathrm{z}): 437[\mathrm{M}+\mathrm{H}]^{+}$. Anal. calcd. for $\mathrm{C}_{23} \mathrm{H}_{20} \mathrm{~N}_{2} \mathrm{O}_{5} \mathrm{~S}: \mathrm{C}, 63.29 ; \mathrm{H}, 4.62 ; \mathrm{N}, 6.42$. Found: C, 63.31; H, 4.77; N, 6.51\%.

(E)-1-[3-(3-(4-hydroxyphenyl) acryloyl) phenyl]-3-tosylurea (4h): Colour: Yellow. Yield: 89\%. M.p.: 185.7 o C. FT-IR (KBr, $\left.V_{\max }, \mathrm{cm}^{-1}\right): 3415,3350(\mathrm{~N}-\mathrm{H}) 3057(\mathrm{C}-\mathrm{H}), 1616(\mathrm{C}=0), 1583$ $(\mathrm{C}=\mathrm{C}), 1555(\mathrm{CONH}), 1517$ (N-H bend), $1337(\mathrm{C}-\mathrm{N}) 1304\left(\mathrm{SO}_{2}\right.$, asym.), $1174\left(\mathrm{SO}_{2}\right.$, sym.). ${ }^{1 \mathrm{H}} \mathrm{NMR}\left(400 \mathrm{MHz}\right.$, DMSO- $\left.d_{6}, \delta, \mathrm{ppm}\right)$ : $2.30\left(\mathrm{~s}, 3 \mathrm{H}, \mathrm{CH}_{3}\right), 7.43(\mathrm{~d}, J=8.1 \mathrm{~Hz}, 3 \mathrm{H}, \mathrm{Ar}-\mathrm{H}), 7.66(\mathrm{~d}, J=15.2$ $\mathrm{Hz}, 1 \mathrm{H}, \mathrm{HC}=\mathrm{CH}(\mathrm{H}-\alpha)), 7.67-8.12(\mathrm{~m}, 7 \mathrm{H}, \mathrm{Ar}-\mathrm{H}), 7.86(\mathrm{~d}, J=15.2$ $\mathrm{Hz}, 1 \mathrm{H}, \mathrm{HC}=\mathrm{CH}(\mathrm{H}-\beta)), 7.87(\mathrm{~d}, J=8.1 \mathrm{~Hz}, 2 \mathrm{H}, \mathrm{Ar}-\mathrm{H}), 9.43(\mathrm{~s}, 1 \mathrm{H}$, $\mathrm{OH}), 9.45(\mathrm{~s}, 1 \mathrm{H}, \mathrm{NH}), 11.93(\mathrm{~s}, 1 \mathrm{H}, \mathrm{NH})$. ESI-MS $(\mathrm{m} / \mathrm{z}): 437$ $[\mathrm{M}+\mathrm{H}]^{+}$. Anal. calcd. for $\mathrm{C}_{23} \mathrm{H}_{20} \mathrm{~N}_{2} \mathrm{O}_{5} \mathrm{~S}: \mathrm{C}, 63.29 ; \mathrm{H}, 4.62 ; \mathrm{N}, 6.42$. Found: C, 63.32; $\mathrm{H}, 4.72 ; \mathrm{N}, 6.55 \%$.

(E)-1-[3-(3-(3-ethoxy-4-hydroxyphenyl) acryloyl) phenyl]-3tosylurea (4i): Colour: Yellow. Yield: 89\%. M.p.: 174.3 'C. FT-IR $\left(\mathrm{KBr}, v_{\max }, \mathrm{cm}^{-1}\right): 3381,3346(\mathrm{~N}-\mathrm{H}), 3092(\mathrm{C}-\mathrm{H}), 1713(\mathrm{C}=0)$, $1664(\mathrm{C}=\mathrm{C}), 1595$ (CONH), 1536 (N-H bend), $1423(\mathrm{C}-\mathrm{N}), 1299$ $\left(\mathrm{SO}_{2}\right.$, asym.), 1090 ( $\mathrm{SO}_{2}$, sym.). ${ }^{1} \mathrm{H}$ NMR (400 MHz, DMSO-d 6 , $\delta$, ppm): $2.30\left(\mathrm{~s}, 3 \mathrm{H}, \mathrm{CH}_{3}\right), 6.54\left(\mathrm{~s}, 2 \mathrm{H}, \mathrm{CH}_{2}\right), 6.82\left(\mathrm{~s}, 3 \mathrm{H}, \mathrm{CH}_{3}\right)$, 6.97-7.34 (m, 7H, Ar-H), 7.42 (d, J = 8.1 Hz, 2H, Ar-H), 7.69 (d, $J$ $=15.4 \mathrm{~Hz}, 1 \mathrm{H}, \mathrm{HC}=\mathrm{CH}(\mathrm{H}-\alpha)$ ), $7.82(\mathrm{~d}, J=8.1 \mathrm{~Hz}, 2 \mathrm{H}, \mathrm{Ar}-\mathrm{H}), 7.94$ $(\mathrm{d}, J=15.4 \mathrm{~Hz}, 1 \mathrm{H}, \mathrm{HC}=\mathrm{CH}(\mathrm{H}-\beta)), 8.78(\mathrm{~s}, 1 \mathrm{H}, \mathrm{OH}), 10.45(\mathrm{~s}, 1 \mathrm{H}$, $\mathrm{NH}), 11.66(\mathrm{~s}, 1 \mathrm{H}, \mathrm{NH})$. ESI-MS $(\mathrm{m} / \mathrm{z}): 481[\mathrm{M}+\mathrm{H}]^{+}$. Anal. calcd. for $\mathrm{C}_{25} \mathrm{H}_{24} \mathrm{~N}_{2} \mathrm{O}_{6} \mathrm{~S}$ : C, 62.49; $\mathrm{H}, 5.03$; N, 5.83. Found: $\mathrm{C}, 62.58 ; \mathrm{H}$, $5.12 ; \mathrm{N}, 5.89 \%$.

(E)-1-[3-(3-(3-methoxy-4-hydroxyphenyl) acryloyl) phenyl]3-tosylurea (4j): Colour: Yellow. Yield: $93 \%$. M.p.: $178.6^{\circ} \mathrm{C}$. FTIR (KBr, $\left.v_{\max }, \mathrm{cm}^{-1}\right): 3367,3304(\mathrm{~N}-\mathrm{H}), 3058(\mathrm{C}-\mathrm{H}), 1726$ (C=0), $1659(\mathrm{C}=\mathrm{C}), 1592(\mathrm{CONH}), 1532$ (N-H bend), $1345(\mathrm{C}-\mathrm{N}), 1313$ ( $\mathrm{SO}_{2}$, asym.), 1157 ( $\mathrm{SO}_{2}$, sym.). ${ }^{1} \mathrm{H}$ NMR $\left(400 \mathrm{MHz}\right.$, DMSO-d $d_{6} \delta$, ppm): $2.30\left(\mathrm{~s}, 3 \mathrm{H}, \mathrm{CH}_{3}\right), 3.96\left(\mathrm{~s}, 3 \mathrm{H}, \mathrm{OCH}_{3}\right), 6.85-7.45(\mathrm{~m}, 7 \mathrm{H}$, Ar-H), 7.42 (d, $J=8.1 \mathrm{~Hz}, 2 \mathrm{H}, \mathrm{Ar}-\mathrm{H}), 7.87$ (d, $J=8.1 \mathrm{~Hz}, 2 \mathrm{H}, \mathrm{Ar}-$ H), $7.68(\mathrm{~d}, J=15.2 \mathrm{~Hz}, 1 \mathrm{H}, \mathrm{HC}=\mathrm{CH}(\mathrm{H}-\alpha)), 7.96(\mathrm{~d}, J=15.2 \mathrm{~Hz}$, $1 \mathrm{H}, \mathrm{HC}=\mathrm{CH}(\mathrm{H}-\beta)), 8.43(\mathrm{~s}, 1 \mathrm{H}, \mathrm{OH}), 9.28(\mathrm{~s}, 1 \mathrm{H}, \mathrm{NH}), 10.87(\mathrm{~s}$, $1 \mathrm{H}, \quad \mathrm{NH})$. ESI-MS $(\mathrm{m} / \mathrm{z}): 467[\mathrm{M}+\mathrm{H}]^{+}$. Anal. calcd. for $\mathrm{C}_{24} \mathrm{H}_{22} \mathrm{~N}_{2} \mathrm{O}_{6} \mathrm{~S}$ : C, 61.79; H, 4.75; N, 6.00. Found: C, 61.65; H, 4.66; $\mathrm{N}, 6.11 \%$.

(E)-1-[3-(3-(2-nitrophenyl) acryloyl) phenyl]-3-tosylurea (4k): Colour: Yellow. Yield: 86\%. M.p.: $231.8{ }^{\circ} \mathrm{C}$. FT-IR (KBr, $\left.V_{\max } \mathrm{cm}^{-1}\right): 3471,3242(\mathrm{~N}-\mathrm{H}), 3092(\mathrm{C}-\mathrm{H}), 1708(\mathrm{C}=\mathrm{O}), 1650$ $(\mathrm{C}=\mathrm{C}), 1586(\mathrm{CONH}), 1528$ (N-H bend), $1349(\mathrm{C}-\mathrm{N}), 1304\left(\mathrm{SO}_{2}\right.$, asym.), 1158 ( $\mathrm{SO}_{2}$, sym.). ${ }^{1} \mathrm{H}$ NMR ( $400 \mathrm{MHz}$, DMSO- $\left.d_{6}, \delta, \mathrm{ppm}\right)$ : $2.30\left(\mathrm{~s}, 3 \mathrm{H}, \mathrm{CH}_{3}\right), 6.95-7.18(\mathrm{~m}, 8 \mathrm{H}, \mathrm{Ar}-\mathrm{H}), 7.41(\mathrm{~d}, J=8.1 \mathrm{~Hz}, 2 \mathrm{H}$, Ar-H), $7.69(\mathrm{~d}, J=15.2 \mathrm{~Hz}, 1 \mathrm{H}, \mathrm{HC}=\mathrm{CH}(\mathrm{H}-\alpha)$ ), 7.87 (d, $J=8.1 \mathrm{~Hz}$, $2 \mathrm{H}, \mathrm{Ar}-\mathrm{H}), 7.95(\mathrm{~d}, J=15.2 \mathrm{~Hz}, 1 \mathrm{H}, \mathrm{HC}=\mathrm{CH}(\mathrm{H}-\beta)), 9.22(\mathrm{~s}, 1 \mathrm{H}$, $\mathrm{NH}), 10.55$ (s, 1H, NH). ESI-MS (m/z): $466[\mathrm{M}+\mathrm{H}]^{+}$. Anal. calcd. for $\mathrm{C}_{23} \mathrm{H}_{19} \mathrm{~N}_{3} \mathrm{O}_{6} \mathrm{~S}$ : C, 59.35; $\mathrm{H}, 4.11 ; \mathrm{N}, 9.03$. Found: $\mathrm{C}, 59.32 ; \mathrm{H}$, 4.16; N, 9.21\%.

(E)-1-[3-(3-(3-nitrophenyl) acryloyl) phenyl]-3-tosylurea (41): Colour: Yellow. Yield: 89\%. M.p.: $172.2{ }^{\circ} \mathrm{C}$. FT-IR (KBr, $v_{\max }$, $\left.\mathrm{cm}^{-1}\right): 3413,3263(\mathrm{~N}-\mathrm{H}), 3063(\mathrm{C}-\mathrm{H}), 1689(\mathrm{C}=0), 1625(\mathrm{C}=\mathrm{C})$, 1585 (CONH), 1521 (N-H bend), 1342 (C-N), 1315 ( $\mathrm{SO}_{2}$, asym.), $1161\left(\mathrm{SO}_{2}\right.$, sym.). ${ }^{1} \mathrm{H}$ NMR $\left(400 \mathrm{MHz}, \mathrm{DMSO}-d_{6}, \delta, \mathrm{ppm}\right): 2.30(\mathrm{~s}$, $\left.3 \mathrm{H}, \mathrm{CH}_{3}\right), 6.95-7.41(\mathrm{~m}, 8 \mathrm{H}, \mathrm{Ar}-\mathrm{H}), 7.43(\mathrm{~d}, J=8.1 \mathrm{~Hz}, 2 \mathrm{H}, \mathrm{Ar}-\mathrm{H})$, $7.67(\mathrm{~d}, J=15.2 \mathrm{~Hz}, 1 \mathrm{H}, \mathrm{HC}=\mathrm{CH}(\mathrm{H}-\alpha)), 7.78(\mathrm{~d}, J=8.1 \mathrm{~Hz}, 2 \mathrm{H}$, Ar-H), 7.91 (d, $J=15.2 \mathrm{~Hz}, 1 \mathrm{H}, \mathrm{HC}=\mathrm{CH}(\mathrm{H}-\beta)), 10.97$ (s, 1H, NH), $11.94(\mathrm{~s}, 1 \mathrm{H}, \mathrm{NH})$. ESI-MS $(\mathrm{m} / \mathrm{z}): 466[\mathrm{M}+\mathrm{H}]^{+}$. Anal. calcd. for $\mathrm{C}_{23} \mathrm{H}_{19} \mathrm{~N}_{3} \mathrm{O}_{6} \mathrm{~S}: \mathrm{C}, 59.35 ; \mathrm{H}, 4.11 ; \mathrm{N}, 9.03$. Found: C, 59.32; H, 4.23; N, 9.21\%.

(E)-1-[3-(3-(5-hydroxy-2-nitrophenyl) acryloyl) phenyl]-3tosylurea (4m): Colour: Yellow. Yield: $85 \%$. M.p.: $166.2{ }^{\circ} \mathrm{C}$. FTIR (KBr, $\left.v_{\max }, \mathrm{cm}^{-1}\right): 3383,3290(\mathrm{~N}-\mathrm{H}), 3068(\mathrm{C}-\mathrm{H}), 1649(\mathrm{C}=0)$, $1614(\mathrm{C}=\mathrm{C}), 1581$ (CONH) 1547 (N-H bend), 1346 (C-N), 1306 ( $\mathrm{SO}_{2}$, asym.), $1156\left(\mathrm{SO}_{2}\right.$, sym.). ${ }^{1} \mathrm{H}$ NMR $\left(400 \mathrm{MHz}, \mathrm{DMSO}-d_{6}, \delta\right.$, ppm): $2.30\left(\mathrm{~s}, 3 \mathrm{H}, \mathrm{CH}_{3}\right), 6.86-7.23(\mathrm{~m}, 7 \mathrm{H}, \mathrm{Ar}-\mathrm{H}), 7.42(\mathrm{~d}, J=8.1$ $\mathrm{Hz}, 2 \mathrm{H}, \mathrm{Ar}-\mathrm{H}), 7.70(\mathrm{~d}, J=15.2 \mathrm{~Hz}, 1 \mathrm{H}, \mathrm{HC}=\mathrm{CH}(\mathrm{H}-\alpha)$ ), $7.83(\mathrm{~d}, J=$ $8.1 \mathrm{~Hz}, 2 \mathrm{H}, \mathrm{Ar}-\mathrm{H}), 7.88(\mathrm{~d}, J=15.2 \mathrm{~Hz}, 1 \mathrm{H}, \mathrm{HC}=\mathrm{CH}(\mathrm{H}-\beta)), 8.42$ (s, 1H, OH), $10.41(\mathrm{~s}, 1 \mathrm{H}, \mathrm{NH}), 11.72(\mathrm{~s}, 1 \mathrm{H}, \mathrm{NH})$. ESI-MS $(\mathrm{m} / \mathrm{z})$ : $482[\mathrm{M}+\mathrm{H}]^{+}$. Anal. calcd. for $\mathrm{C}_{23} \mathrm{H}_{19} \mathrm{~N}_{3} \mathrm{O}_{7} \mathrm{~S}: \mathrm{C}, 57.37 ; \mathrm{H}, 3.98 ; \mathrm{N}$, 8.73. Found: C, $57.44 ; \mathrm{H}, 3.78 ; \mathrm{N}, 8.24 \%$.

(E)-1-[3-(3-(3-fluorophenyl) acryloyl) phenyl]-3-tosylurea (4n): Colour: Yellow. Yield: 84\%. M.p.: $183.5{ }^{\circ} \mathrm{C}$. FT-IR (KBr, $\left.V_{\max }, \mathrm{cm}^{-1}\right): 3241(\mathrm{~N}-\mathrm{H}), 3029(\mathrm{C}-\mathrm{H}), 1704(\mathrm{C}=0), 1647(\mathrm{C}=\mathrm{C})$, 1591 (CONH), 1522 (N-H bend), 1344 (C-N), 1294 ( $\mathrm{SO}_{2}$, asym.), $1160\left(\mathrm{SO}_{2}\right.$, sym.). ${ }^{1} \mathrm{H}$ NMR $\left(400 \mathrm{MHz}, \mathrm{DMSO}-d_{6}, \delta, \mathrm{ppm}\right): 2.30(\mathrm{~s}$, $\left.3 \mathrm{H}, \mathrm{CH}_{3}\right), 7.18-7.97(\mathrm{~m}, 8 \mathrm{H}, \mathrm{Ar}-\mathrm{H}), 7.41$ (d, $\left.J=8.1 \mathrm{~Hz}, 2 \mathrm{H}, \mathrm{Ar}-\mathrm{H}\right)$, $7.66(\mathrm{~d}, J=15.4 \mathrm{~Hz}, 1 \mathrm{H}, \mathrm{HC}=\mathrm{CH}(\mathrm{H}-\alpha)), 7.88(\mathrm{~d}, J=8.1 \mathrm{~Hz}, 2 \mathrm{H}$, Ar-H), 8.03 (d, $J=15.4 \mathrm{~Hz}, 1 \mathrm{H}, \mathrm{HC}=\mathrm{CH}(\mathrm{H}-\beta)), 10.03$ (s, 1H, NH), $11.16(\mathrm{~s}, 1 \mathrm{H}, \mathrm{NH})$. ESI-MS $(\mathrm{m} / \mathrm{z}): 439[\mathrm{M}+\mathrm{H}]^{+}$. Anal. calcd. for $\mathrm{C}_{23} \mathrm{H}_{19} \mathrm{FN}_{2} \mathrm{O}_{4} \mathrm{~S}: \mathrm{C}, 63.00 ; \mathrm{H}, 4.37$; N, 6.39. Found: C, 63.12; $\mathrm{H}$, $4.44 ; \mathrm{N}, 6.43 \%$.

(E)-1-[3-(3-(4-fluorophenyl) acryloyl) phenyl]-3-tosylurea (4o): Colour: Yellow. Yield: $87 \%$. M.p.: $150.3{ }^{\circ} \mathrm{C}$. FT-IR $(\mathrm{KBr}$, $\left.v_{\max } \mathrm{cm}^{-1}\right)$ : 3383, $3289(\mathrm{~N}-\mathrm{H}), 3068(\mathrm{C}-\mathrm{H}), 1649(\mathrm{C}=0), 1615$ $(\mathrm{C}=\mathrm{C}), 1581(\mathrm{CONH}), 1512$ (N-H bend), $1346(\mathrm{C}-\mathrm{N}), 1306\left(\mathrm{SO}_{2}\right.$, asym.), $1156\left(\mathrm{SO}_{2}\right.$, sym.). ${ }^{1} \mathrm{H}$ NMR $\left(400 \mathrm{MHz}\right.$, DMSO- $\left.d_{6}, \delta, \mathrm{ppm}\right)$ : $2.30\left(\mathrm{~s}, 3 \mathrm{H}, \mathrm{CH}_{3}\right), 7.05-7.68(\mathrm{~m}, 8 \mathrm{H}, \mathrm{Ar}-\mathrm{H}), 7.69(\mathrm{~d}, J=15.2 \mathrm{~Hz}$, $1 \mathrm{H}, \mathrm{HC}=\mathrm{CH}(\mathrm{H}-\alpha)), 7.79(\mathrm{~d}, J=8.1 \mathrm{~Hz}, 2 \mathrm{H}, \mathrm{Ar}-\mathrm{H}), 7.42(\mathrm{~d}, J=8.1$ $\mathrm{Hz}, 2 \mathrm{H}, \mathrm{Ar}-\mathrm{H}), 7.99$ (d, $J=15.2 \mathrm{~Hz}, 1 \mathrm{H}, \mathrm{HC}=\mathrm{CH}(\mathrm{H}-\beta)$ ), 9.94 (s, 
1H, NH), $11.01(\mathrm{~s}, 1 \mathrm{H}, \mathrm{NH})$. ESI-MS $(\mathrm{m} / \mathrm{z}): 439[\mathrm{M}+\mathrm{H}]^{+}$. Anal. calcd. for $\mathrm{C}_{23} \mathrm{H}_{19} \mathrm{FN}_{2} \mathrm{O}_{4} \mathrm{~S}$ : C, 63.00; $\mathrm{H}, 4.37$; N, 6.39. Found: C, 63.12; H, 4.24; N, 6.32\%.

(E)-1-[3-(3-(2-chlorophenyl) acryloyl) phenyl]-3-tosylurea (4p): Colour: Yellow. Yield: 88\%. M.p.: 244.5 ' $\mathrm{C}$. FT-IR (KBr, $\left.V_{\max } \mathrm{cm}^{-1}\right): 3225(\mathrm{~N}-\mathrm{H}), 3071(\mathrm{C}-\mathrm{H}), 1716(\mathrm{C}=0), 1695(\mathrm{C}=\mathrm{C})$, 1684 (CONH), 1558 (N-H bend), 1340 (C-N), 1300 ( $\mathrm{SO}_{2}$, asym.), 1153 ( $\mathrm{SO}_{2}$, sym.). ${ }^{1} \mathrm{H}$ NMR (400 MHz, DMSO- $\left.d_{6}, \delta, \mathrm{ppm}\right): 2.30$ (s, $\left.3 \mathrm{H}, \mathrm{CH}_{3}\right), 6.66-7.15(\mathrm{~m}, 8 \mathrm{H}, \mathrm{Ar}-\mathrm{H}), 7.41(\mathrm{~d}, J=8.1 \mathrm{~Hz}, 2 \mathrm{H}, \mathrm{Ar}-\mathrm{H})$, $7.66(\mathrm{~d}, J=15.4 \mathrm{~Hz}, 1 \mathrm{H}, \mathrm{HC}=\mathrm{CH}(\mathrm{H}-\alpha)), 7.83(\mathrm{~d}, J=8.1 \mathrm{~Hz}, 2 \mathrm{H}$, $\mathrm{Ar}-\mathrm{H}), 8.05(\mathrm{~d}, J=15.4 \mathrm{~Hz}, 1 \mathrm{H}, \mathrm{HC}=\mathrm{CH}(\mathrm{H}-\beta)), 10.89(\mathrm{~s}, 1 \mathrm{H}, \mathrm{NH})$, $12.13(\mathrm{~s}, 1 \mathrm{H}, \mathrm{NH})$. ESI-MS $(\mathrm{m} / \mathrm{z}): 455[\mathrm{M}+\mathrm{H}]^{+}$. Anal. calcd. for $\mathrm{C}_{23} \mathrm{H}_{19} \mathrm{ClN}_{2} \mathrm{O}_{4} \mathrm{~S}$ : C, 60.72; H, 4.21; N, 6.16. Found: C, 60.65; H, 4.32; $\mathrm{N}, 6.14 \%$.

(E)-1-[3-(3-(4-chlorophenyl) acryloyl) phenyl]-3-tosylurea (4q): Colour: Yellow. Yield: 92\%. M.p.: $227.5{ }^{\circ} \mathrm{C}$. FT-IR (KBr, $\left.v_{\max } \mathrm{cm}^{-1}\right): 3450,3340(\mathrm{~N}-\mathrm{H}), 3049(\mathrm{C}-\mathrm{H}), 1650(\mathrm{C}=0), 1626$ $(\mathrm{C}=\mathrm{C}), 1588(\mathrm{CONH}), 1566$ (N-H bend), $1341(\mathrm{C}-\mathrm{N}), 1270\left(\mathrm{SO}_{2}\right.$, asym.), $1133\left(\mathrm{SO}_{2}\right.$, sym.). ${ }^{1} \mathrm{H}$ NMR $\left(400 \mathrm{MHz}\right.$, DMSO- $\left.d_{6}, \delta, \mathrm{ppm}\right)$ : $2.30\left(\mathrm{~s}, 3 \mathrm{H}, \mathrm{CH}_{3}\right), 7.38(\mathrm{~d}, J=8.1 \mathrm{~Hz}, 2 \mathrm{H}, \mathrm{Ar}-\mathrm{H}), 7.45-7.63(\mathrm{~m}, 8 \mathrm{H}$, $\mathrm{Ar}-\mathrm{H}), 7.67(\mathrm{~d}, J=15.2 \mathrm{~Hz}, 1 \mathrm{H}, \mathrm{HC}=\mathrm{CH}(\mathrm{H}-\alpha)), 7.88(\mathrm{~d}, J=8.1 \mathrm{~Hz}$, $2 \mathrm{H}, \mathrm{Ar}-\mathrm{H}), 7.90$ (d, J = $15.2 \mathrm{~Hz}, 1 \mathrm{H}, \mathrm{HC}=\mathrm{CH}(\mathrm{H}-\beta)), 9.73$ (s, 1H, $\mathrm{NH}), 11.21(\mathrm{~s}, 1 \mathrm{H}, \mathrm{NH})$. ESI-MS $(\mathrm{m} / \mathrm{z}): 455[\mathrm{M}+\mathrm{H}]^{+}$. Anal. calcd. for $\mathrm{C}_{23} \mathrm{H}_{19} \mathrm{ClN}_{2} \mathrm{O}_{4} \mathrm{~S}: \mathrm{C}, 60.72 ; \mathrm{H}, 4.21 ; \mathrm{N}, 6.16$. Found: C, 60.65; H, $4.37 ; \mathrm{N}, 6.15 \%$.

(E)-1-[3-(3-(2,4-dichlorophenyl) acryloyl) phenyl]-3tosylurea (4r): Colour: Yellow. Yield: 85\%. M.p.: 220.5 'C. FT-IR (KBr, $\left.v_{\max }, \mathrm{cm}^{-1}\right)$ : 3454, $3345(\mathrm{~N}-\mathrm{H}), 3069(\mathrm{C}-\mathrm{H}), 1708(\mathrm{C}=0)$, $1633(\mathrm{C}=\mathrm{C}), 1617$ (CONH), 1584 (N-H bend), 1359 (C-N) 1270 ( $\mathrm{SO}_{2}$, asym.), 1135 ( $\mathrm{SO}_{2}$, sym.). ${ }^{1} \mathrm{H}$ NMR ( $400 \mathrm{MHz}, \mathrm{DMSO}-d_{6}, \delta$, ppm): 2.30 (s, 3H, $\left.\mathrm{CH}_{3}\right), 6.66-7.35(\mathrm{~m}, 7 \mathrm{H}, \mathrm{Ar}-\mathrm{H}), 7.44(\mathrm{~d}, J=8.1$ $\mathrm{Hz}, 2 \mathrm{H}, \mathrm{Ar}-\mathrm{H}), 7.66$ (d, $J=15.4 \mathrm{~Hz}, 1 \mathrm{H}, \mathrm{HC}=\mathrm{CH}(\mathrm{H}-\alpha)$ ), $7.74(\mathrm{~d}, J=$ $8.1 \mathrm{~Hz}, 2 \mathrm{H}, \mathrm{Ar}-\mathrm{H}), 7.88$ (d, $J=15.4 \mathrm{~Hz}, 1 \mathrm{H}, \mathrm{HC}=\mathrm{CH}(\mathrm{H}-\beta)), 9.68$ (s, 1H, NH), $10.91(\mathrm{~s}, 1 \mathrm{H}, \mathrm{NH})$. ESI-MS (m/z): $490[\mathrm{M}+\mathrm{H}]^{+}$. Anal. calcd. for $\mathrm{C}_{23} \mathrm{H}_{18} \mathrm{Cl}_{2} \mathrm{~N}_{2} \mathrm{O}_{4} \mathrm{~S}$ : C, 56.45; H, 3.71; N, 5.72. Found: C, 56.53; H, 3.82; N, 5.86\%.

(E)-1-[3-(3-(3-bromophenyl) acryloyl) phenyl]-3-tosylurea (4s): Colour: Yellow. Yield: 84\%. M.p.: 214.2 ${ }^{\circ} \mathrm{C}$. FT-IR (KBr, $\left.V_{\max } \mathrm{cm}^{-1}\right): 3445,3349(\mathrm{~N}-\mathrm{H}), 3068(\mathrm{C}-\mathrm{H}), 1642(\mathrm{C}=0), 1596$ $(\mathrm{C}=\mathrm{C}), 1511(\mathrm{CONH}), 1447$ (N-H bend), $1334(\mathrm{C}-\mathrm{N}), 1306\left(\mathrm{SO}_{2}\right.$, asym.), $1168\left(\mathrm{SO}_{2}\right.$, sym.). ${ }^{1} \mathrm{H}$ NMR $\left(400 \mathrm{MHz}\right.$, DMSO- $\left.d_{6}, \delta, \mathrm{ppm}\right)$ : $2.30\left(\mathrm{~s}, 3 \mathrm{H}, \mathrm{CH}_{3}\right), 7.35-7.68(\mathrm{~m}, 8 \mathrm{H}, \mathrm{Ar}-\mathrm{H}), 7.41(\mathrm{~d}, J=8.1 \mathrm{~Hz}, 2 \mathrm{H}$, $\mathrm{Ar}-\mathrm{H}), 7.69(\mathrm{~d}, J=15.4 \mathrm{~Hz}, 1 \mathrm{H}, \mathrm{HC}=\mathrm{CH}(\mathrm{H}-\alpha)), 7.78(\mathrm{~d}, J=8.1 \mathrm{~Hz}$, 2H, Ar-H), 7.98 (d, J = 15.4 Hz, 1H, HC=CH (H- $\beta$ )), 9.12 (s, 1H, $\mathrm{NH}), 8.99(\mathrm{~s}, 1 \mathrm{H}, \mathrm{NH})$. ESI-MS $(\mathrm{m} / \mathrm{z}): 500[\mathrm{M}+\mathrm{H}]^{+}$. Anal. calcd. for $\mathrm{C}_{23} \mathrm{H}_{19} \mathrm{BrN}_{2} \mathrm{O}_{4} \mathrm{~S}: \mathrm{C}, 55.32 ; \mathrm{H}, 3.83$; N, 5.61. Found: C, 55.28; H, $3.82 ; \mathrm{N}, 5.55 \%$.

(E)-1-[3-(3-(4-bromophenyl) acryloyl) phenyl]-3-tosylurea (4t): Colour: Yellow. Yield: 81\%. M.p.: $244.2{ }^{\circ} \mathrm{C}$. FT-IR (KBr, $v_{\max }$, $\mathrm{cm}^{-1}$ ): 3439, $3349(\mathrm{~N}-\mathrm{H}), 3019(\mathrm{C}-\mathrm{H}), 1639$ (C=0), 1579 (C=C), 1513 (CONH), 1442 (N-H bend), 1329 (C-N), 1312 ( $\mathrm{SO}_{2}$, asym.), 1177 ( $\mathrm{SO}_{2}$, sym.). ${ }^{1} \mathrm{H}$ NMR (400 MHz, DMSO- $\left.d_{6}, \delta, \mathrm{ppm}\right): 2.30$ (s, $\left.3 \mathrm{H}, \mathrm{CH}_{3}\right), 7.33-7.61(\mathrm{~m}, 8 \mathrm{H}, \mathrm{Ar}-\mathrm{H}), 7.44(\mathrm{~d}, J=8.1 \mathrm{~Hz}, 2 \mathrm{H}, \mathrm{Ar}-\mathrm{H})$, $7.67(\mathrm{~d}, J=15.4 \mathrm{~Hz}, 1 \mathrm{H}, \mathrm{HC}=\mathrm{CH}(\mathrm{H}-\alpha)), 7.72(\mathrm{~d}, J=8.1 \mathrm{~Hz}, 2 \mathrm{H}$, $\mathrm{Ar}-\mathrm{H}), 7.98(\mathrm{~d}, J=15.4 \mathrm{~Hz}, 1 \mathrm{H}, \mathrm{HC}=\mathrm{CH}(\mathrm{H}-\beta)), 9.11(\mathrm{~s}, 1 \mathrm{H}, \mathrm{NH})$, $8.99(\mathrm{~s}, 1 \mathrm{H}, \mathrm{NH})$. ESI-MS $(\mathrm{m} / \mathrm{z}): 500[\mathrm{M}+\mathrm{H}]^{+}$. Anal. calcd. for $\mathrm{C}_{23} \mathrm{H}_{19} \mathrm{BrN}_{2} \mathrm{O}_{4} \mathrm{~S}$ : C, 55.32; H, 3.83; N, 5.61. Found: C, 55.22; $\mathrm{H}$, $3.81 ; \mathrm{N}, 5.52 \%$.

(E)-1-[3-(3-(4-allyloxyphenyl) acryloyl) phenyl]-3-tosylurea (4u): Colour: Yellow. Yield: 85\%. M.p.: $162.2{ }^{\circ} \mathrm{C}$. FT-IR (KBr, $\left.V_{\max } \mathrm{cm}^{-1}\right): 3328,3227(\mathrm{~N}-\mathrm{H}), 3058(\mathrm{C}-\mathrm{H}), 1632(\mathrm{C}=\mathrm{O}), 1588$ $(\mathrm{C}=\mathrm{C}), 1502(\mathrm{CONH}), 1458\left(\mathrm{~N}-\mathrm{H}\right.$ bend), $1326(\mathrm{C}-\mathrm{N}), 1281\left(\mathrm{SO}_{2}\right.$, asym.), 1127 ( $\mathrm{SO}_{2}$, sym.). ${ }^{1 \mathrm{H}} \mathrm{NMR}\left(400 \mathrm{MHz}\right.$, DMSO- $\left.d_{6}, \delta, \mathrm{ppm}\right)$ : $2.30\left(\mathrm{~s}, 3 \mathrm{H}, \mathrm{CH}_{3}\right), 4.49\left(\mathrm{~s}, 2 \mathrm{H}, \mathrm{CH}_{2}\right), 5.33\left(\mathrm{~s}, 2 \mathrm{H}, \mathrm{CH}_{2}\right), 5.51(\mathrm{~s}, 1 \mathrm{H}$, $\mathrm{CH}), 7.42(\mathrm{~d}, J=8.1 \mathrm{~Hz}, 2 \mathrm{H}, \mathrm{Ar}-\mathrm{H}), 7.67-7.91(\mathrm{~m}, 8 \mathrm{H}, \mathrm{Ar}-\mathrm{H}), 7.83$ (d, $J=8.1 \mathrm{~Hz}, 2 \mathrm{H}, \mathrm{Ar}-\mathrm{H}), 7.87(\mathrm{~d}, J=15.2 \mathrm{~Hz}, 1 \mathrm{H}, \mathrm{HC}=\mathrm{CH}(\mathrm{H}-\alpha)$ ), $8.04(\mathrm{~d}, J=15.2 \mathrm{~Hz}, 1 \mathrm{H}, \mathrm{HC}=\mathrm{CH}(\mathrm{H}-\beta)), 8.98(\mathrm{~s}, 1 \mathrm{H}, \mathrm{NH}), 9.96$ (s, $1 \mathrm{H}, \quad \mathrm{NH})$. ESI-MS $(\mathrm{m} / \mathrm{z}): 477 \quad[\mathrm{M}+\mathrm{H}]^{+}$. Anal. calcd. for $\mathrm{C}_{26} \mathrm{H}_{24} \mathrm{~N}_{2} \mathrm{O}_{5} \mathrm{~S}$ : C, 65.53; H, 5.08; N, 5.88. Found: C, 65.34; H, 5.90; $\mathrm{N}, 5.75 \%$.
(E)-1-[3-(3-(Phenylethene-yl) acryloyl) phenyl]-3-tosylurea (4v): Colour: Yellow. Yield: 94\%. M.p.: 178.3 oC. FT-IR (KBr, $\left.v_{\max } \mathrm{cm}^{-1}\right)$ : 3443, $3297(\mathrm{~N}-\mathrm{H}), 3060(\mathrm{C}-\mathrm{H}), 1630(\mathrm{C}=0), 1573$ $(\mathrm{C}=\mathrm{C}), 1509$ (CONH), 1447 (N-H bend), $1354(\mathrm{C}-\mathrm{N}), 1301\left(\mathrm{SO}_{2}\right.$, asym.), 1141 ( $\mathrm{SO}_{2}$, sym.). ${ }^{1} \mathrm{H}$ NMR ( $400 \mathrm{MHz}$, DMSO- $\left.d_{6}, \delta, \mathrm{ppm}\right)$ : 2.30 (s, 3H, $\left.\mathrm{CH}_{3}\right), 7.33-7.12(\mathrm{~m}, 11 \mathrm{H}, \mathrm{Ar}-\mathrm{H}), 7.42(\mathrm{~d}, J=8.1 \mathrm{~Hz}$, $2 \mathrm{H}, \mathrm{Ar}-\mathrm{H}), 7.68(\mathrm{~d}, J=15.4 \mathrm{~Hz}, 1 \mathrm{H}, \mathrm{HC}=\mathrm{CH}(\mathrm{H}-\alpha)), 7.78(\mathrm{~d}, J=8.1$

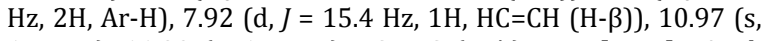
$1 \mathrm{H}, \mathrm{NH}), 11.99(\mathrm{~s}, 1 \mathrm{H}, \mathrm{NH})$. ESI-MS $(\mathrm{m} / \mathrm{z}): 447[\mathrm{M}+\mathrm{H}]^{+}$. Anal. calcd. for $\mathrm{C}_{25} \mathrm{H}_{22} \mathrm{~N}_{2} \mathrm{O}_{4} \mathrm{~S}$ : C, 67.25; $\mathrm{H}, 4.97 ; \mathrm{N}, 6.27$. Found: C, 67.18; H, 4.12; N, 6.23\%.

(E)-1-[3-(3-(pyridine-3-yl) acryloyl) phenyl]-3-tosylurea (4w): Colour: Yellow. Yield: 86\%. M.p.: 231.8 oC. FT-IR (KBr, $\left.V_{\max } \mathrm{cm}^{-1}\right): 3423,3218(\mathrm{~N}-\mathrm{H}), 3016(\mathrm{C}-\mathrm{H}), 1686(\mathrm{C}=0), 1650$ $(\mathrm{C}=\mathrm{C}), 1593(\mathrm{CONH}), 1520$ (N-H bend), $1352(\mathrm{C}-\mathrm{N}), 1303\left(\mathrm{SO}_{2}\right.$, asym.), 1146 ( $\mathrm{SO}_{2}$, sym.). ${ }^{1} \mathrm{H}$ NMR $\left(400 \mathrm{MHz}\right.$, DMSO- $\left.d_{6}, \delta, \mathrm{ppm}\right)$ : $2.30\left(\mathrm{~s}, 3 \mathrm{H}, \mathrm{CH}_{3}\right), 6.97-7.54(\mathrm{~m}, 8 \mathrm{H}, \mathrm{Ar}-\mathrm{H}), 7.41(\mathrm{~d}, J=8.1 \mathrm{~Hz}, 2 \mathrm{H}$, $\mathrm{Ar}-\mathrm{H}), 7.68(\mathrm{~d}, J=15.4 \mathrm{~Hz}, 1 \mathrm{H}, \mathrm{HC}=\mathrm{CH}(\mathrm{H}-\alpha)), 7.88(\mathrm{~d}, J=8.1 \mathrm{~Hz}$, $2 \mathrm{H}, \mathrm{Ar}-\mathrm{H}), 7.93(\mathrm{~d}, J=15.4 \mathrm{~Hz}, 1 \mathrm{H}, \mathrm{HC}=\mathrm{CH}(\mathrm{H}-\beta)), 10.45(\mathrm{~s}, 1 \mathrm{H}$, $\mathrm{NH}), 11.99$ (s, 1H, NH). ESI-MS (m/z): $422[\mathrm{M}+\mathrm{H}]^{+}$. Anal. calcd. for $\mathrm{C}_{22} \mathrm{H}_{19} \mathrm{~N}_{3} \mathrm{O}_{4} \mathrm{~S}: \mathrm{C}, 62.69 ; \mathrm{H}, 4.54 ; \mathrm{N}, 9.97$. Found: $\mathrm{C}, 62.69 ; \mathrm{H}$, $4.62 ; \mathrm{N}, 9.78 \%$.

(E)-1-[3-(3-(pyridine-4-yl) acryloyl) phenyl]-3-tosylurea (4x): Colour: Yellow. Yield: $89 \%$. M.p.: $188.0{ }^{\circ} \mathrm{C}$. FT-IR (KBr, $\left.V_{\max } \mathrm{cm}^{-1}\right): 3388,3277(\mathrm{~N}-\mathrm{H}), 2887(\mathrm{C}-\mathrm{H}), 1649(\mathrm{C}=\mathrm{O}), 1620$ $(\mathrm{C}=\mathrm{C}), 1586(\mathrm{CONH}), 1498(\mathrm{~N}-\mathrm{H}$ bend $), 1346(\mathrm{C}-\mathrm{N}), 1296\left(\mathrm{SO}_{2}\right.$, asym.), 1156 ( $\mathrm{SO}_{2}$, sym.). ${ }^{1} \mathrm{H}$ NMR ( $400 \mathrm{MHz}$, DMSO- $\left.d_{6}, \delta, \mathrm{ppm}\right)$ : 2.30 (s, 3H, CH $), 7.42(\mathrm{~d}, J=8.1 \mathrm{~Hz}, 2 \mathrm{H}, \mathrm{Ar}-\mathrm{H}), 7.53-7.81(\mathrm{~m}, 8 \mathrm{H}$, $\mathrm{Ar}-\mathrm{H}), 7.66(\mathrm{~d}, J=15.2 \mathrm{~Hz}, 1 \mathrm{H}, \mathrm{HC}=\mathrm{CH}(\mathrm{H}-\alpha)), 7.79(\mathrm{~d}, J=8.1 \mathrm{~Hz}$, $2 \mathrm{H}, \mathrm{Ar}-\mathrm{H}$ ), 7.89 (d, $J=15.2 \mathrm{~Hz}, 1 \mathrm{H}, \mathrm{HC}=\mathrm{CH}(\mathrm{H}-\beta)), 10.42$ (s, 1H, $\mathrm{NH}), 11.64(\mathrm{~s}, 1 \mathrm{H}, \mathrm{NH})$. ESI-MS $(\mathrm{m} / \mathrm{z}): 422[\mathrm{M}+\mathrm{H}]^{+}$. Anal. calcd. for $\mathrm{C}_{22} \mathrm{H}_{19} \mathrm{~N}_{3} \mathrm{O}_{4} \mathrm{~S}: \mathrm{C}, 62.69 ; \mathrm{H}, 4.54 ; \mathrm{N}, 9.97$. Found: $\mathrm{C}, 62.71 ; \mathrm{H}$, 4.66; N, 9.88\%.

(E)-1-[3-(3-(Anthracen-9-yl) acryloyl) phenyl]-3-tosylurea (4y): Colour: Yellow. Yield: 93\%. M.p.: $174.4{ }^{\circ} \mathrm{C}$. FT-IR (KBr, $\left.V_{\max } \mathrm{cm}^{-1}\right): 3298,3242(\mathrm{~N}-\mathrm{H}), 2887(\mathrm{C}-\mathrm{H}), 1694 \mathrm{~m}(\mathrm{C}=\mathrm{O}), 1600$ $(\mathrm{C}=\mathrm{C}), 1537(\mathrm{CONH}), 1452$ (N-H bend), $1343(\mathrm{C}-\mathrm{N}), 1319\left(\mathrm{SO}_{2}\right.$, asym.), 1157 ( $\mathrm{SO}_{2}$, sym.). ${ }^{1 \mathrm{H}} \mathrm{NMR}\left(400 \mathrm{MHz}\right.$, DMSO- $\left.d_{6}, \delta, \mathrm{ppm}\right)$ : $2.30\left(\mathrm{~s}, 3 \mathrm{H}, \mathrm{CH}_{3}\right), 7.31-7.54(\mathrm{~m}, 13 \mathrm{H}, \mathrm{Ar}-\mathrm{H}), 7.42(\mathrm{~d}, J=8.1 \mathrm{~Hz}$, $2 \mathrm{H}, \mathrm{Ar}-\mathrm{H}), 7.73(\mathrm{~d}, J=15.2 \mathrm{~Hz}, 1 \mathrm{H}, \mathrm{HC}=\mathrm{CH}(\mathrm{H}-\alpha)), 7.81(\mathrm{~d}, J=8.1$ $\mathrm{Hz}, 2 \mathrm{H}, \mathrm{Ar}-\mathrm{H}), 8.11$ (d, $J=15.2 \mathrm{~Hz}, 1 \mathrm{H}, \mathrm{HC}=\mathrm{CH}(\mathrm{H}-\beta)$ ), 10.78 (s, $1 \mathrm{H}, \mathrm{NH}), 12.11(\mathrm{~s}, 1 \mathrm{H}, \mathrm{NH})$. ESI-MS $(\mathrm{m} / \mathrm{z}): 521[\mathrm{M}+\mathrm{H}]^{+}$. Anal. calcd. for $\mathrm{C}_{31} \mathrm{H}_{24} \mathrm{~N}_{2} \mathrm{O}_{4} \mathrm{~S}$ : C, 71.52; $\mathrm{H}, 4.65 ; \mathrm{N}, 5.38$. Found: C, 71.48; H, 4.53; N, 5.25\%.

\subsection{Pharmacological activity}

The 5-LO inhibitory potential of the synthesized compounds $(\mathbf{4 a - 4 y )}$ was determined by 5 -LO inhibition assay (UV-Kinetic method) as described by Sircar et al. [40]. For the evaluation of 5-LO inhibitory activity, the enzymatic activity of 5-LO was measured spectrophotometrically using potato 5-LO [41] and an incubation mixture containing $80 \mathrm{mM}$ linoleic acid and $50 \mathrm{mM}$ sodium phosphate buffer $(\mathrm{pH}=6.3)$. The reaction was initiated by the addition of an enzyme buffer mix to substrate (Linoleic acid) and the enzyme activity was monitored as an increase in rate of absorbance at $234 \mathrm{~nm}$ on a UV/visible spectrophotometer (Varion Cary-50 UV-Visible spectrophotometer) for 120 sec. Each experiment was conducted by incubating along with control at various concentrations of the test substances with enzyme buffer mix for $2 \mathrm{~min}$ before addition of the substrate. The percentage inhibition was calculated by comparing slope or increase in absorbance of test substance with that of control enzyme activity. The assay was performed in triplicate and mean values were used for the calculation. The $\mathrm{IC}_{50}$ values were obtained using fenny probed analysis software. The result for the test compound was compared with the positive control abietic acid (LI01020) [42]. The results of 5-LO inhibitory activity are given in Table 1. 
Table 1. Physical characterization and 5-LO inhibitory activity data of diarylsulfonylurea-chalcone hybrids $\mathbf{4 a - 4 y}$ produced via Scheme 1.

\begin{tabular}{|c|c|c|c|c|c|c|}
\hline Compound & $\mathbf{R}$ & Yield a (\%) & Molecular weight (g) & Molecular formula & M.p. $\left({ }^{\circ} \mathrm{C}\right)$ & $\begin{array}{l}\mathrm{IC}_{50}(\mu \mathrm{g} / \mathrm{mL}) \\
(\mathrm{mean} \pm \mathrm{SEM}) \mathrm{c}\end{array}$ \\
\hline $4 \mathrm{a}$ & $\mathrm{C}_{6} \mathrm{H}_{5}$ & 97 & 420 & $\mathrm{C}_{23} \mathrm{H}_{20} \mathrm{~N}_{2} \mathrm{O}_{4} \mathrm{~S}$ & 151.3 & $38.66 \pm 0.25$ \\
\hline $4 b$ & $4-\mathrm{MeC}_{6} \mathrm{H}_{4}$ & 89 & 434 & $\mathrm{C}_{24} \mathrm{H}_{22} \mathrm{~N}_{2} \mathrm{O}_{4} \mathrm{~S}$ & 233.8 & $25.24 \pm 0.45$ \\
\hline $4 \mathrm{c}$ & $4-\mathrm{NMe}_{2} \mathrm{C}_{6} \mathrm{H}_{4}$ & 88 & 463 & $\mathrm{C}_{25} \mathrm{H}_{25} \mathrm{~N}_{3} \mathrm{O}_{4} \mathrm{~S}$ & 150.5 & $35.11 \pm 0.23$ \\
\hline $4 d$ & 2,4-diOMeC ${ }_{6} \mathrm{H}_{3}$ & 84 & 480 & $\mathrm{C}_{25} \mathrm{H}_{24} \mathrm{~N}_{2} \mathrm{O}_{6} \mathrm{~S}$ & 163.3 & $23.11 \pm 0.32$ \\
\hline $4 \mathrm{e}$ & $3,4,5$-triOMeC ${ }_{6} \mathrm{H}_{2}$ & 88 & 510 & $\mathrm{C}_{26} \mathrm{H}_{26} \mathrm{~N}_{2} \mathrm{O}_{7} \mathrm{~S}$ & 198.6 & $22.18 \pm 0.11$ \\
\hline $4 \mathrm{f}$ & $2-\mathrm{OHC}_{6} \mathrm{H}_{4}$ & 86 & 436 & $\mathrm{C}_{23} \mathrm{H}_{20} \mathrm{~N}_{2} \mathrm{O}_{5} \mathrm{~S}$ & 258.9 & $35.13 \pm 0.45$ \\
\hline $4 \mathrm{~g}$ & $3-\mathrm{OHC}_{6} \mathrm{H}_{4}$ & 82 & 436 & $\mathrm{C}_{23} \mathrm{H}_{20} \mathrm{~N}_{2} \mathrm{O}_{5} \mathrm{~S}$ & 185.2 & $46.22 \pm 0.12$ \\
\hline $4 \mathrm{~h}$ & $4-\mathrm{OHC}_{6} \mathrm{H}_{4}$ & 89 & 436 & $\mathrm{C}_{23} \mathrm{H}_{20} \mathrm{~N}_{2} \mathrm{O}_{5} \mathrm{~S}$ & 185.7 & $39.24 \pm 0.34$ \\
\hline $4 \mathrm{i}$ & $3-\mathrm{OEt}, 4-\mathrm{OHC}_{6} \mathrm{H}_{3}$ & 89 & 480 & $\mathrm{C}_{25} \mathrm{H}_{24} \mathrm{~N}_{2} \mathrm{O}_{6} \mathrm{~S}$ & 174.3 & $26.31 \pm 0.52$ \\
\hline $4 j$ & $3-\mathrm{OMe}, 4-\mathrm{OHC}_{6} \mathrm{H}_{3}$ & 93 & 466 & $\mathrm{C}_{24} \mathrm{H}_{22} \mathrm{~N}_{2} \mathrm{O}_{6} \mathrm{~S}$ & 178.6 & $22.18 \pm 0.17$ \\
\hline $4 \mathrm{k}$ & $2-\mathrm{NO}_{2} \mathrm{C}_{6} \mathrm{H}_{4}$ & 86 & 465 & $\mathrm{C}_{23} \mathrm{H}_{19} \mathrm{~N}_{3} \mathrm{O}_{6} \mathrm{~S}$ & 231.8 & $24.28 \pm 0.13$ \\
\hline 41 & $3-\mathrm{NO}_{2} \mathrm{C}_{6} \mathrm{H}_{4}$ & 89 & 465 & $\mathrm{C}_{23} \mathrm{H}_{19} \mathrm{~N}_{3} \mathrm{O}_{6} \mathrm{~S}$ & 172.2 & $33.66 \pm 0.61$ \\
\hline $4 \mathrm{~m}$ & $5-\mathrm{OH}, 2-\mathrm{NO}_{2} \mathrm{C}_{6} \mathrm{H}_{3}$ & 85 & 481 & $\mathrm{C}_{23} \mathrm{H}_{19} \mathrm{~N}_{3} \mathrm{O}_{7} \mathrm{~S}$ & 166.2 & $44.18 \pm 0.53$ \\
\hline $4 n$ & $3-\mathrm{FC}_{6} \mathrm{H}_{4}$ & 84 & 438 & $\mathrm{C}_{23} \mathrm{H}_{19} \mathrm{FN}_{2} \mathrm{O}_{4} \mathrm{~S}$ & 183.5 & $18.12 \pm 0.42$ \\
\hline 40 & $4-\mathrm{FC}_{6} \mathrm{H}_{4}$ & 87 & 438 & $\mathrm{C}_{23} \mathrm{H}_{19} \mathrm{FN}_{2} \mathrm{O}_{4} \mathrm{~S}$ & 150.3 & $11.77 \pm 0.21$ \\
\hline $4 p$ & $2-\mathrm{ClC}_{6} \mathrm{H}_{4}$ & 88 & 454 & $\mathrm{C}_{23} \mathrm{H}_{19} \mathrm{ClN}_{2} \mathrm{O}_{4} \mathrm{~S}$ & 244.5 & $24.81 \pm 0.51$ \\
\hline $4 q$ & $4-\mathrm{ClC}_{6} \mathrm{H}_{4}$ & 92 & 454 & $\mathrm{C}_{23} \mathrm{H}_{19} \mathrm{ClN}_{2} \mathrm{O}_{4} \mathrm{~S}$ & 227.5 & $15.32 \pm 0.16$ \\
\hline $4 r$ & 2,4-diClC $6 \mathrm{H}_{3}$ & 85 & 489 & $\mathrm{C}_{23} \mathrm{H}_{18} \mathrm{Cl}_{2} \mathrm{~N}_{2} \mathrm{O}_{4} \mathrm{~S}$ & 220.5 & $7.88 \pm 0.14$ \\
\hline $4 s$ & $3-\mathrm{BrC}_{6} \mathrm{H}_{4}$ & 84 & 499 & $\mathrm{C}_{23} \mathrm{H}_{19} \mathrm{BrN}_{2} \mathrm{O}_{4} \mathrm{~S}$ & 214.2 & $29.41 \pm 0.27$ \\
\hline $4 \mathrm{t}$ & $4-\mathrm{BrC}_{6} \mathrm{H}_{4}$ & 81 & 499 & $\mathrm{C}_{23} \mathrm{H}_{19} \mathrm{BrN}_{2} \mathrm{O}_{4} \mathrm{~S}$ & 244.2 & $18.12 \pm 0.32$ \\
\hline $4 u$ & 4-Allyl- $\mathrm{OC}_{6} \mathrm{H}_{4}$ & 85 & 476 & $\mathrm{C}_{26} \mathrm{H}_{24} \mathrm{~N}_{2} \mathrm{O}_{5} \mathrm{~S}$ & 162.2 & $29.13 \pm 0.23$ \\
\hline $4 \mathrm{v}$ & Phenylethene-yl & 94 & 446 & $\mathrm{C}_{25} \mathrm{H}_{22} \mathrm{~N}_{2} \mathrm{O}_{4} \mathrm{~S}$ & 178.3 & $44.38 \pm 0.13$ \\
\hline $4 w$ & Pyridin-3-yl & 86 & 421 & $\mathrm{C}_{22} \mathrm{H}_{19} \mathrm{~N}_{3} \mathrm{O}_{4} \mathrm{~S}$ & 231.8 & $41.22 \pm 0.49$ \\
\hline $4 \mathrm{x}$ & Pyridin-4-yl & 89 & 421 & $\mathrm{C}_{22} \mathrm{H}_{19} \mathrm{~N}_{3} \mathrm{O}_{4} \mathrm{~S}$ & 188.0 & $33.31 \pm 0.22$ \\
\hline $4 y$ & Anthracen-9-yl & 93 & 520 & $\mathrm{C}_{31} \mathrm{H}_{24} \mathrm{~N}_{2} \mathrm{O}_{4} \mathrm{~S}$ & 174.4 & $14.91 \pm 0.77$ \\
\hline Standard b & - & - & - & - & - & $4.34 \pm 0.37$ \\
\hline
\end{tabular}

a Crystallization solvent is ethanol.

b Abietic acid (LI01020).

c SEM = Standard error of the mean.

\section{Results and discussion}

\subsection{Synthesis}

The reaction sequence employed in the synthesis of diarylsulfonylurea-chalcone hybrids (4a-4y) is shown in the Scheme 1 and their physical properties are depicted in Table 1. The key intermediate in the present study 1-(3-acetylphenyl)3-tosylurea (3) was synthesized by reaction of 3aminoacetophenone (1) with methylchloroformate under basic conditions at $0{ }^{\circ} \mathrm{C}$ temperature to give methyl-3-acetylphenyl carbamate (2) followed by the reaction with toluene sulphonamide and on the other hand subsequent ClaisenSchmidt condensation of the intermediate (3) with appropriate aromatic/heteroaromatic aldehydes in ethanolic $\mathrm{KOH}$ solution to give the corresponding diarylsulfonylurea-chalcone hybrids (4a-4y) in good yield (Scheme 1). All the structures of the compounds were appropriately established by spectroscopic data and analytical methods.

\subsection{5-Lipoxygenase inhibitory activity}

The investigation of in vitro 5-LO inhibitory activity screening data (Table 1) revealed that the compounds $\mathbf{4 r}$ and 40 demonstrated comparatively the most potent inhibitory activity, with $\mathrm{IC}_{50}$ values of $7.88 \pm 0.14 \mu \mathrm{g} / \mathrm{mL}$ and $11.77 \pm 0.21$ $\mu \mathrm{g} / \mathrm{mL}$, respectively. It is interesting to note that the compounds 4y, 4q, 4t and 4n also showed appreciable inhibitory activity with $\mathrm{IC}_{50}$ values of $14.91 \pm 0.77,15.32 \pm 0.16$, $18.12 \pm 0.32$ and $18.12 \pm 0.42 \mu \mathrm{g} / \mathrm{mL}$, respectively. The other compounds such as $\mathbf{4 b}, \mathbf{4 d}, \mathbf{4 i - 4 l}, \mathbf{4 p}, \mathbf{4 s}, \mathbf{4 u}$ and $\mathbf{4 x}$ showed moderate level of activity at concentrations ( $\mathrm{IC}_{50}$ ) ranging from $22.18 \pm 0.11$ to $33.31 \pm 0.22 \mu \mathrm{g} / \mathrm{mL}$. The compounds $\mathbf{4 a}, \mathbf{4 c}, \mathbf{4 f}-\mathbf{h}$, $\mathbf{4 m}, \mathbf{4 v}$ and $\mathbf{4 w}$ exhibited comparatively less activity with $\mathrm{IC}_{50}$ values ranging from $35.11 \pm 0.23$ to $46.22 \pm 0.12 \mu \mathrm{g} / \mathrm{mL}$ in comparison with the standard drug (Abietic acid (LI01020), $\mathrm{IC}_{50}: 4.34 \pm 0.37 \mu \mathrm{g} / \mathrm{mL}$ ).

A close look at the SAR (Structure-Activity Relationship) of these compounds clearly exhibited the inherent phenomenon of 5-LO inhibitory activity associated with the basic skeleton consisting of diarylsulfonylurea and $\alpha, \beta$-unsaturated ketone moieties as seen in case of the unsubstituted compound $\mathbf{4 a}$ with $\mathrm{IC}_{50}$ value of $38.66 \pm 0.25 \mu \mathrm{g} / \mathrm{mL}$, which in some cases was enhanced by the influence of some substituents and decreased by some other substituents. For example, the compounds $\mathbf{4 r}$

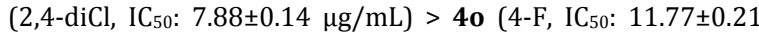
$\mu \mathrm{g} / \mathrm{mL})>4 \mathbf{q}\left(4-\mathrm{Cl}, \mathrm{IC}_{50}: 15.32 \pm 0.16 \mu \mathrm{g} / \mathrm{mL}\right)>4$ n $\left(3-\mathrm{F}, \mathrm{IC}_{50}\right.$ : $18.12 \pm 0.42 \mu \mathrm{g} / \mathrm{mL})>\mathbf{4 t}\left(4-\mathrm{Br}, \mathrm{IC}_{50}: 18.12 \pm 0.32 \mu \mathrm{g} / \mathrm{mL}\right)>\mathbf{4 p}$ (2-Cl, IC $50: 24.81 \pm 0.51 \mu \mathrm{g} / \mathrm{mL})>4 s\left(3-\mathrm{NH}_{2}, \mathrm{IC}_{50}: 29.41 \pm 0.27\right.$ $\mu \mathrm{g} / \mathrm{mL}$ ) having halogen substituents either at ortho or meta or para positions significantly enhanced the activity. A reduction in the activity was observed when the substituted phenyl ring B was replaced by a cinnamyl moiety, as seen in the case of compound $4 \mathbf{v}\left(\mathrm{IC}_{50}\right.$ value $44.38 \pm 0.13 \mu \mathrm{g} / \mathrm{mL}$ ). The presence of a 3 -pyridyl ring in compound $\mathbf{4} \mathbf{w}$ in the place of substituted phenyl ring $B$ of $\alpha, \beta$ - unsaturated carbonyl system enhanced the activity compared to the one possessing cinnamyl moiety, but less than that of the one having substituted phenyl ring. It is also interesting to see the presence of 4-pyridyl ring in the place of substituted phenyl ring B contributed to an increase in activity compared to the one possessing 3-pyridyl ring, respectively as seen in the case of compounds $\mathbf{4 x}$ and $\mathbf{4} \mathbf{w}$ with $\mathrm{IC}_{50}$ values $33.31 \pm 0.22$ and $41.22 \pm 0.49 \mu \mathrm{g} / \mathrm{mL}$, respectively. It was observed that the replacement of substituted phenyl ring B with allyloxy group at para position enhanced 5-LO inhibitory activity $\left(4 \mathbf{u}, \mathrm{IC}_{50}: 29.13 \pm 0.23 \mu \mathrm{g} / \mathrm{mL}\right)$. The presence of a 9 anthracenyl ring in compound $\mathbf{4 y}\left(\mathrm{IC}_{50}: 14.91 \pm 0.77 \mu \mathrm{g} / \mathrm{mL}\right.$ ) in the place of substituted phenyl ring $B$ of $\alpha, \beta$ - unsaturated carbonyl system significantly increased the activity compared to the one possessing 3-pyridyl and 4-pyridyl ring systems.

However, it was noticed that various aromatic/heteroaromatic rings substituted at position 3 of $\alpha, \beta$-unsaturated carbonyl system followed its activity order as anthreacen-9-yl > pyridin-4-yl > phenyl > pyridin-3-yl moieties, respectively. It was also noted that the compounds substituted with electron releasing groups was found to be biologically relevant and the activity order was $\left(4 \mathrm{e}\left(3,4,5\right.\right.$-tri- $\left.\mathrm{OCH}_{3}, \mathrm{IC}_{50}: 22.18 \pm 0.11 \mu \mathrm{g} / \mathrm{mL}\right)$ $>$ 4d $\left(2,4-d i-\mathrm{OCH}_{3}, \mathrm{IC}_{50}: 23.11 \pm 0.32 \pm 0.23 \mu \mathrm{g} / \mathrm{mL}\right)>4 \mathbf{b}\left(4-\mathrm{CH}_{3}\right.$, $\left.\mathrm{IC}_{50}: 25.24 \pm 0.45 \mu \mathrm{g} / \mathrm{mL}\right)>4 \mathrm{c}\left(4-\mathrm{N}\left(\mathrm{CH}_{3}\right)_{2}, \mathrm{IC}_{50}: 35.11 \pm 0.23\right.$ $\mu \mathrm{g} / \mathrm{mL})$ ), respectively. It is important that less activity was observed when the hydroxyl groups are substituted at different positions on the phenyl ring as seen in the case of compounds $\mathbf{4 f}-\mathbf{4 h}$ and the order of activity was $\mathbf{4 f}\left(2-\mathrm{OH}, \mathrm{IC}_{50}: 35.13 \pm 0.45\right.$ $\mu \mathrm{g} / \mathrm{mL})>\mathbf{4 h}\left(4-\mathrm{OH}, \mathrm{IC}_{50}: 39.24 \pm 0.34 \mu \mathrm{g} / \mathrm{mL}\right)>\mathbf{4 g}\left(3-\mathrm{OH}, \mathrm{IC}_{50}\right.$ : 
$44.18 \pm 0.53 \mu \mathrm{g} / \mathrm{mL}$ ) respectively. The compounds $4 \mathbf{j}$ (IC 50 : $22.18 \pm 0.17 \mu \mathrm{g} / \mathrm{mL}$ ) having methoxyl group at substitution on the phenyl ring B at position 3, 4i ( $\mathrm{IC}_{50}: 26.31 \pm 0.52 \mu \mathrm{g} / \mathrm{mL}$ ) having ethoxy group at substitution on the phenyl ring $\mathrm{B}$ at position 3 and $\mathbf{4 m}$ ( $\mathrm{IC}_{50}: 44.18 \pm 0.53 \mu \mathrm{g} / \mathrm{mL}$ ) having nitro group at substitution on the phenyl ring $\mathrm{B}$ at position 2 along with the hydroxyl group substitution at positions 4 (in case of $\mathbf{4 j}$ and $\mathbf{4 i}$ ) and 5 (in case of $\mathbf{4 m}$ ), respectively showed enhanced level of 5LO inhibitory activity when compared with that of the compounds (4f-4h) possessing only hydroxyl group substitution. It is notable that enhanced level of activity was observed when the nitro group introduced on the phenyl ring $B$ of $\alpha, \beta$-unsaturated carbonyl system at 2 and 3 positions as seen in the case of compounds $\mathbf{4 k}$ and $\mathbf{4 1}$ with $\mathrm{IC}_{50}$ values $24.28 \pm 0.13$ and $33.66 \pm 0.6 \mu \mathrm{g} / \mathrm{mL}$, respectively.

\section{Conclusion}

In summary, we synthesized and characterized a series of diarylsulfonylurea-chalcone hybrids (4a-4y). For the first time, this class of compounds were screened for 5-LO inhibitory activity and the results revealed the positive contribution of halogen substituents on the phenyl ring $B$ of $\alpha, \beta$-unsaturated ketone towards the observed 5-LO inhibitory activity. The observed activity may also be due to diarylsulfonylurea and $\alpha, \beta$-unsaturated ketone moieties forming part of the basic structure of these molecules. The results indicated that further development of such compounds might be of biological interest.

\section{Acknowledgements}

One of the authors (Bharat Kumar) is thankful to Gitam University, Visakhapatnam, for providing necessary facilities to carry out research work.

\section{References}

[1]. Kenyon, V.; Chorny, I.; Carvajal, W. J.; Holman, T. R.; Jacobson, M.P. J. Med. Chem. 2006, 49, 1356-1363.

[2]. Werz, O. Curr. Drug Targets Inflamm. Allergy. 2002, 1, 23-44

[3]. Babu, M. A.; Shakya, N.; Prathipati, P.; Kaskhedikara, S. G.; Saxena, A. K. Bioorgan. Med. Chem. 2002, 10, 4035-4041.

[4]. Zhang, H. B.; Zhang, Y. A.; Wu, G. Z.; Zhou, J. P.; Huang, W. L.; Hu, X. W. Bioorg. Med. Chem. Lett. 2009, 19, 1740-1744.

[5]. Frezza, M.; Soulere, L.; Reverchon, S.; Guiliani, N.; Jerez, C.; Queneau, Y.; Doutheau, A. Bioorg. Med. Chem. Lett. 2008, 16, 3550-3556.

[6]. Winters, M. P.; Crysler, C.; Subasinghe, N.; Ryan, D.; Leong, L.; Zhao, S.; Donatelli, R.; Yurkow, E.; Mazzulla, M.; Boczon, L.; Manthey, C. L.; Molloy, C.; Raymond, H.; Murray, L.; McAlonan, L.; Tomczuka, B. Bioorg. Med. Chem. Lett. 2008, 18, 1926-1930.

[7]. Leon, C.; Rodrigues, J.; Dominguez, N. G. D.; Charris, J.; Gut, J.; Rosenthal, P. J.; Dominguez, J. N. Eur. J. Med. Chem. 2007, 42, 735-742.

[8]. Ameya, A.; Chavan, C.; Nandini, R. P. J. Chin. Chem. Soc. 2007, 54, 771777.

[9]. Hanson, J.; Dogne, J. M.; Ghiotto, J.; Moray, A. L.; Kinsella, B. T.; Pirotte, B. J. Med. Chem. 2007, 50, 3928-3936.

[10]. Lehr, P.; Billich, A.; Wolff, B.; Nussbaumer, P. Bioorg. Med. Chem. Lett. 2005, 15, 1235-1238.

[11]. Salamon, E.; Mannhold, R.; Weber, H.; Lemoine, H.; Frank, W. J. Med. Chem. 2002, 45, 1086-1089.

[12]. Heitsch, H.; Becker, A. H. R.; Kleemann, W. H.; Wagner, A. Bioorgan. Med. Chem. 1997, 5, 673-678.

[13]. Toth, E. J.; Grindey, B. G.; Ehlhardt, J. W.; Ray, E. J.; Boder, G. B.; Bewley, R. J.; Klingerman, K. K.; Gates, B. S.; Rinzel, M. S.; Schultz, R. M.; Weir, L. C.; Worzalla, F. J. J. Med. Chem. 1997, 40, 1018-1025.

[14]. Picard, A. J.; Obrien, M. P.; Sliskovic, R. D.; Anderson, K. M.; Bousley, F. R.; Hamelehle, L. K.; Krause, R. B.; Stanfield, L. R. J. Med. Chem. 1996, 39, 1243-1252.

[15]. Khelili, S.; Leclerc, G.; Faury, G.; Verdetti, J. Bioorgan. Med. Chem. 1995, 5, 495-503.

[16]. Lee, C. J. M.; Elberling, A. J.; Nagasawa, T. H. J. Med. Chem. 1992, 35, 3641-3647.

[17]. Mohamadi, F.; Spees, M. M.; Grindey, B. G. J. Med. Chem. 1992, 35, 3012-3016.

[18]. Love, B.; Snader, M. K. J. Med. Chem. 1968, 2, 1251-1270.

[19]. Uehling, E. D.; Donaldson, H. K.; Deaton, N. D.; Hyman, E. C.; Sugg, E. E.; Barrett, G. D.; Hughes, G. R.; Reitter, B.; Adkison, K. K.; Lancaster, E. M.;
Lee, F.; Hart, R.; Paulik, A. M.; Sherman, W. B.; True, T.; Cowan, C. J. Med. Chem. 2002, 45, 567-583.

[20]. Choi, J. K.; Noh, M. K.; Choi, D. J.; Park, S. J.; Won, S. H.; Kim, R. J.; Kim, S J.; Yoon, M. Y. B Korean Chem. Soc. 2006, 10, 1697-1700.

[21]. Shuichi, F.; Masanori, I.; Atsutaka, Y.; Makoto, M.; Morihiro, M.; Iichiro, S. J. Biol. Chem. 2005, 24, 23653-23659.

[22]. Dinkova, K. A. T.; Massiah, M. A.; Bozak, R. E.; Hicks, R. J.; Talalay P. P. Natl. Acad. Sci. USA 2001, 98, 3404-3409.

[23]. Nakagawa, G.; Lee, K. Tetrahedron Lett. 2006, 47, 8263-8265.

[24]. Narender, T.; Shweta; Gupta S. Bioorg. Med. Chem. Lett. 2004, 14(15), 3913-3916.

[25]. Nielsen, S. F.; Chen, M.; Theander, T. G.; Kharazmi, A.; Christensen, S. B Bioorg. Med. Chem. Lett. 1995, 5, 449-452.

[26]. Wu, J. H.; Wang, X. H.; Lee, K. H. Bioorgan. Med. Chem. 2003, 13, 1813 1815.

[27]. Wu, X.; Wilairat, P.; Go, M. Bioorg. Med. Chem. Lett. 2002, 12, 22992302.

[28]. Phrutivorapongkul, A.; Kirtikaro, K. Chem. Pharm. Bull. 2003, 51(6), 746-748.

[29]. Kumar, S. K.; Hager, E.; Pettit, C.; Gurulingappa, H.; Davidson, N. E.; Khan, S. R. J. Med. Chem. 2003, 46, 2813-2815.

[30]. Narender, T.; Khaliq, T.; Shweta, N.; Gupta, S. Bioorgan. Med. Chem. 2005, 13, 6543-6550.

[31]. Nakaido, T.; Ohmoto, T. Chem. Pharm. Bull. 1984, 32(12), 4929-4934.

[32]. Kostanekki, S. T.; Szabranki, W. Ber. Dtsch. Chem. Ges. 1904, 37, 2634 2635.

[33]. Jun, N.; Hong, G.; Jun, K. Bioorgan. Med. Chem. 2007, 15(6), 2396-2402.

[34]. Zhao, L. M.; Jin, H. S.; Sun, L. P.; Piao, H. R.; Quan, Z. S. Bioorg. Med. Chem. Lett. 2005, 15(22), 5027-5029.

[35]. Assia, S.; Kaddour, L.; Mahieddine, M.; Nouara, Z.; Paul, M. Eur. J. Chem. 2011, 2(3), 311-313.

[36]. Mohammed, R. A.; Mohammed, H. R. K.; Vedula, G. S.; Nasreen, B.; Syed, A.; Yejella, R. P. Eur. J. Chem. 2012, 3(2), 186-190.

[37]. Mohammed, S. B.; Aly, A. A.; Ashraf, F. W.; Marwa, M. R. Eur. J. Chem. 2013, 4(2), 92-97.

[38]. Avupati, V. R.; Yejella, R. P.; Guntuku, G.; Gunta, P. Bioorg. Med. Chem. Lett. 2012, 22, 1031-1035.

[39]. Claisen, L.; Claparede A. Ber. 1881, 14, 2463-2479.

[40]. Sircar, J. C.; Shwender, C. J.; Johnson, E. A. Prostaglandins 1983, 25, 393-396.

[41]. Reddenna, P.; Whelan, J.; Maddipati, R. K. R. Methods Enzymol. 1990 $187,268-277$.

[42]. Ulusu, N. N.; Ercil, D.; Sakar, M. K.; Tezcan, E. E. Phytother. Res. 2002, 16, 88-90. 